\title{
Spotted Hyena Optimization and Simulated Annealing-based NLOS Nodes Localization Scheme for Improving Warning Message Dessimination in VANETS
}

\section{Lenin S.B}

Sri Manakula Vinayagar Educational Trust

Narayana Samy Tamilarasan ( $\sim$ neithalarasu@gmail.com )

Sri Indu College of Engineering \& Technology

\section{Research Article}

Keywords: Vehicular Ad hoc Networks (VANETs), Spotted Hyena Optimization, Simulated Annealing, Veins Simulator, NLOS nodes

Posted Date: June 2nd, 2021

DOI: https://doi.org/10.21203/rs.3.rs-462585/v1

License: (c) (i) This work is licensed under a Creative Commons Attribution 4.0 International License.

Read Full License 


\section{$\underline{\text { Title Page }}$}

Title: Spotted Hyena Optimization and Simulated Annealing-based NLOS nodes localization scheme for improving warning message dessimination in VANETs

Author 1

Author 2 and Corresponding Author : Dr. N. Tamilarasan : Professor, Department of ECE, :Sri Indu college of Engineering and Technology, :Hyderabad, Telangana-501 510

:E-mail: neithalarasu@gmail.com

:Phone Number: +91-9843030698 


\section{Spotted Hyena Optimization and Simulated Annealing-based NLOS nodes localization}

\section{scheme for improving warning message dessimination in VANETs}

Abstract-An accurate localization technique is considered as the significant entity in Vehicular Ad hoc Networks (VANETs) for facilitating emergency message data transmission in diversified critical safety applications. In VANETs, the system of global positioing is generally used for estimating the position of the vehicles in the network for attaining neighborhood awareness in the event of warning message dissemination. However, the existence of green foliages, buildings, indoor parking lots and urban streen canyons introduces NLOS situation that introduces unwanted errors that crumbles the degree of data dissemination in emergency situations. In this paper, Spotted Hyena and Simulated Annealing Optimization Algorithm (SHSAOA)-based positioning scheme was proposed for precise estimation of NLOS nodes. It included the advantages of improved simulated annealing (SA) integrated into SHOA for establishing better balance between the process of exploitation and exploration in the search space. This positioning approach generated candidate solutions by deriving the merits of the trajectory-based charateristics of SA throughout the algorithmic development process in order to improve the local optimization process. This proposed SHSAOA utilized the distance infotmation that are associated with the vehicle trajectory, number of vehicles and error in distance information for assessing the precise location of the NLOS nodes in the network. The simulation results of the proposed SHSAOA scheme confirmed minimized localization error with maximized accuracy in transmission, warning message transmission rate, channel utilization degree and neighborhood awareness degree with different vehicular density and NLOS nodes.

Keywords: Vehicular Ad hoc Networks (VANETs), Spotted Hyena Optimization, Simulated Annealing, Veins Simulator, NLOS nodes.

\section{Introduction}

Vehicular Ad hoc Networks (VANETs) is a specific category of mobile ad hoc networks in which the vehicles are equipped with wireless communication devices. This inclusion of wireless devices can directly facilitate the vehicles to cooperate with one another without any kind of centralized control and infrastructure, thereby attaining rapid delivery of data to the applications. This rapid and reliable delivery of data supports a diversified number of intelligent transportation system (ITS) applications that includes safety transport or driver assistance systems. These ITS applications necessitates rapid solution for provisioning accurate services as the key success of these applications targets on efficient and reliable data dissemination. However, the distinct characteristics of vehicle environment need to be considered for achieving efficient data dissemination. A number of factors such as vehicles movement that changes rapidly and frequently influence reliable data dissemination. The vehicular speed is another factor that impacts the wireless signal that in turn results in high intermittent connectivity between the vehicles under interaction. Moreover, density of vehicles which is very high in urban areas and very sparse in rural areas or highway roads also influences the degree of reliable dat dissemination in the network. In specific, Vehicle-to-Vehicle (V2V) communication induces each and every individual vehicles to generate warning message in stimulus to the predicted emergency event identified in a real-time 
scenario. Information for precise localization or positioning during the occurrence of emergency events is essential for supporting the drivers in making reactive decision process that supports the critically safe real-time applications during vehicle accidents. However, identifying and exchanging accurate potential information about the vehicular location during the occurrence of emergency events is determined to be completely challenging. This challenge posed during the exchange of location information under warning message dissemination in vehicular network need to be handled with utmost care in order to prevent the loss of human life.

In general, Global Positioning System (GPS) equipments are incorporated by vehicles for the purpose of accurately positioning the location of each and every individual vehicles with which it is interacting during the emergency situations. However, the location information provisioned by GPS equipments may be inaccurate, when it is influenced by the existence of NLOS situations in the network. The NLOS situations generally occur due to tall trees, green foliages, road intersections, tall trucks and tall buildings in dense urban area that acts as obstacles and hinders the direct interaction between vehicles during emergency message dissemination. The street anyons and indoor packing lots which are devoid of potential satellite visibility in dense urban areas also constitute NLOS conditions. In particular, vehicular communication is prone to signal interference, since they exhibit diversified patterns based on the conditions of the environment in which they move and cooperate with one another. Further, reliable communication is prevented due to the existence of physical objects and construction sites present on the road sides has the maximum probability of introducing interference with the radio signals. This interference of signals by the obstacles in the vehicular environment introduces a NLOS state to the vehicular nodes and induces the drivers to make worst reactive decisions, when they are about to change lanes or merging into a highway. This NLOS state of the vehicular nodes are futher classified into intentional and unintentional category depending on the impact they introduce into the vehicular network during the process of reliable data dissemination. The intentional NLOS node state is visualized, when malicious attacks and fake positions are launched the intruders on the vehicular network. On the other hand, the unintentional node states emerge based on the existence of physical stationary obstacles such as trees, buildings or moving objects like tall trucks in the dense urban area. In specific, the proposed positioning scheme is attributed towards the localization of NLOS nodes that concentrate towards the detection of stationary or mobile obstacles that aids in better and significant dissemination of emergency message in the vehicular network. Moreover, moving obstacles like tall trucks introduce interference and has the possibility of preventing the drivers' visibility from line of sight during the vehicular communication. These dynamic objects like tall trucks are considered as obstacles that come in different shapes, compositions, densities and move in different speeds resulting in additional NLOS conditions. These obstacles influence the process of information exchange and location updating between the neighboring vehicles during warning message dissemination under critical situations. Furthermore, the obstacles hinders the exchange of information about speed, direction and location of individual vehicles during the process of inter-vehicuar communication. This prevention of information sharing by obstacles induces the drivers on the road to take worst reactive decisions that lead to fatal accidents and loss of human life. In this context, a number of multi-hopping approaches were incorporated for propagating warning messages beyond the communication range, hidden NLOS nodes, interference and packet collisions unfortunately terminates data dissemination process. Hence, NLOS nodes need to be localized or positioned with utmost accuracy and fast reactive rate in order to minimize delay which is highly required during the transmission of warning messages. A 
comprehensive protocol that could attribute towards rapid and reliable warning message delivery with minimized delay during emergency situations becomes essential in vehicular network. In addition, the time gap between the generation, transmission and reception of emergency messages need to be minimized by focusing on the objective of establishing and maintaining maximum coverage between the vehicular nodes.

A diversified number of research works over the recent years were contributed to the literature for the purpose of handling the issues that impacts or introduces NLOS situations. The majority of the proposed NLOS localization approaches mainly targeted on the issue of signal strength, authentication, signal blockage, signal interference and communication range. NLOS node positioning or location verification schemes that aids in estimating the vehicular node availability, source message reliability, node position verification and different issues related to message integrity and Quality of Service (QoS) were also been contributed to the literature. In specific, accurate NLOS nodes' localization or positioning schemes are considered to be highly indespensable for attaining reliable warning message delivery rate with minimized delay. These NLOS node localization approaches are categorized into range and range-free positioning schemes based on the inclusion of propagation model principles and distance information. This localization approaches can be implemented only through a cooperative node positioning mechanism or reference nodes of the network. But, cooperative node positioning schemes is considered to be more successful on par with the anchor node-based NLOS positioning scheme as they are capable in exploiting the benefits of $\mathrm{V} 2 \mathrm{~V}$ communication. In particular, the NLOS nodes' neighbours during cooperative node positioning exchange location information and facilitate the option of estimating the distance between each vehiculat nodes and their associated neighbours. Moreover, intelligent metaheuristic cooperative node positioning schemes proposed using Weighted intertiabased dynamic bat algorithm, and Raccom Optimization Algorithm confirmed its suitability and ideality in localizing NLOS nodes during warning message dissemination in vehicular network. The possibility of integrating SHOA with SA algorithms for targeting on the objective of balancing exploitation and exploration during searching process in the population space motivated its utilization in NLOS node positioning process. Moreover, integrated SHOA and SA optimization algorithm in rapid time establishes potential coverage with maximized accuracy for determining the global optimal solution from the search space with its inherited capabilities and memory strength.

In this paper, Spotted Hyena and Simulated Annealing Optimization Algorithm (SHSAOA)-based NLOS node positioning scheme is proposed for determining accurate location of NLOS nodes in order to improve warning message delivery rate with minimized delay in emergency situations. This SHSAOA is proposed for rapidly and reactively localizing the position of NLOS nodes based on the intelligent search mechanism that mimics the characteristic hunting and encircling phenonmenon of spotted hyenas. It is proposed as significant cooperative positioning technique that enhances the optimal solution determined at the end of each individual iteration by remembering them, such that it can utilized for increasing the rapidness in localizing the NLOS nodes in the forthcoming iterations. The simulation experiments of the proposed SHSAOA is conducted using Veins simulator in order to determine its localization capability with respect to the performance metrics of mean emergency message delivery rate, mean neighborhood awareness rate, mean localization rate and localizing error rate with scalable imcrease in the vehicular nodes and reference nodes. 
The major contributions of the proposed SHSAOA is listed as follows:

i) It is proposed as a cooperative localization approach with the intelligent hunting and encircling characteristics of Spotted Hyena search ageny that aids in determining the difference between the previous solutions and the current solutions (location of nodes) for enhancing the efficiency of positioning NLOS nodes in the network.

ii) It offers superior adaptability by adjusting the value associated with the random vector $C_{V(A)}$ and $\overrightarrow{E_{P}}$ for achieving potential swirching between exploration and exploitation during NLOS node localization process.

iii) It included the advantages of improved simulated annealing (SA) integrated into SHOA for establishing better balance between the process of exploitation and exploration in the search space.

iv) The integrated SHOA and GR-ISA algorithm generated candidate solutions by deriving the merits of the trajectory-based charateristics of SA throughout the algorithmic development process in order to improve the local optimization process.

v) It also incorprated the process of reannealing for improving the non-dominated solutions identified in the novel generation phase for increasing the selection pressure towards the Pareto front.

The remaining sections of the paper is structured as follows.Section 2 presents the detailed view of the existing NLOS positioning meta-heuristic algorithms contributed to the literature over the recent years. Section 3 depicts the complete implementation process of the proposed SHSAOA-based NLOS node positioning scheme with the steps involved in the implementation process of SHOA and GR-ISA algorithms for NLOS node localization. Section 4 demonstrates the simulation setup and results of the proposed SHSAOA scheme with proper justification that lie behind their predominant performance. Section 5 concluded the paper with the remarkable contributions of the proposed SHSAOA scheme with future scope of enhancement.

\section{Related work}

In this section, the comprehensive review of the existing NLOS localization approaches are presented with their pros and cons.

An experience and plausibility integrated fuzzy trust model-was proposed for securing the communications that happens between vehicular nodes during the existence of NLOS nodes in the network [16]. This fuzzy trust model was executed with a sequence of security checks that guaranteed the information correctness which are possibly derived from the authorized vehicles. It was evaluated based on the accuracy level of event location derived from the adopted facility of fog nodes. It was proposed with the capability of preventing imprecise, incomplete and inaccurate information collected from vehicles. It was also contributed for mitigating thr interrupting effects imtroduced by the movable and immovable obstacles of the vehicular network. The simulation results was confirmed to detect faulty nodes and malicious attacks by preventing data precision and uncertainity in vehicular network in the existence of line of sight and non-line of sight situations. However, the degree of warning message delivery and reduction in delay incurred 
during warning message dissemination still requires a room of improvement. Then, a distance information based cooperative vehicle localization improvement (DICVLI) scheme was proposed for determining accurate GPS positions of the interacting vehicles during the event of emergency [17]. This localization improvement approach utilized the benefits of extended Kalman filter for executing data fusion that targeted on error minimization based in distance and GPS information. It was proposed with the three dimensional parameters of distance information such as distance information error, vehicle trajectory and number of vehicles for assessing the position accuracy of vehicles cooperating in the network. It also included weighted average method for gaining more confidence about the distance information that are derived from the neighbors, which is in closer promixity to the vehicular nodes under localization. The simulation results of this DICVLI scheme was confirmed to minimize the GPS erroe by $63 \%$, better than the baseline schemes considered for investigation. However, the localization accuracy of this DICVLI scheme was not maximized with increase in the number of obstacles in the urban regions.

Further, Cooperative Volunteer Protocol-based Localization Scheme (CVPLS) was proposed for utilizing the benefits of volunteer nodes termed reference nodes in determining the position of the target vehicles exhibiting NLOS situations [18]. This CVPLS scheme included the merits of context-aware system for classifying the OBU components with the associated interaction for the purpose of sensing data and making decisions. The simulation results of this CVPLS scheme confirmed better performance in term of latency, overhead, channel utilization, neighborhood awareness and packet delivery ratio, compared to the baseline approaches. It was also determined to be predominant in detecting NLOS situations in an efficient and effective way for urban and highway scenario. A Dynamic Virtual Bat Algorithm using Weighted Inertia (VBAWI) was proposed for positioning NLOS nodes with maximized accuracy during the event of warning message dissemination [19]. This VBAWI localization approach included the intensification and extensification capabilities of particle swarm optimization and simulating annealing for potential localization of NLOS nodes. This localization approach prevented the premature convergence issue by utilizing a weighted inertia parameter that reactively increased and decreased the degree of exploitation and exploration depending on the location of NLOS nodes. The results of VBAWI localization approach confirmed specific increase in the potentialty of neighbor awareness rate. However, the delay incurred during warning message delivery is comparatively high compared to the existing works of the literature.

Authentication and Plausibiity-based security model (APSM) was proposed for improving the rapidity in positioning NLOS nodes under emergency situations [20]. This NLOS localizing security model was proposed for handling illegitimate nodes that intentionaly enters into the network for broadcasting fake information about the vehicular nodes position. It included the merits of a probabilistic data structure termed cuckoo filter in order to facilitate space-proficiency with effective identification of location-based fake information exchanged in the network. It also incorporated a fuzzy logic model for estimating the plausibility degree that aids in better handling of the inaccurate information thata are shared in emergency conditions. This included fuzzy logic 
model detected inconsistent data and dealt with intentionally mishaving NLOS nodes that have the probability of introducing non-light of sight conditions into the network. This APSM model was determined to be better in minimizing commuincation overhead and maximizing F-Measure compared to the localization schemes considered for investigation. A Reliable Localization Scheme using Harris Hawk Optimization Algorithm (RLSHHOA) was proposed for guaranteeing reliable warning message delivery under emergency conditions [21]. This RLSHHOA derived the cooperative characteristics and chasing styles of Harris hawks named surprise pounce for achieving better localization through reference nodes. It included the intelligence of HHOA algorithm for determining the position of unknown NLOS nodes from all the directions of the network. It adopted an adaptive localizing mechanism that gets updated based on the dynamic characteristics of NLOS nodes moving in the vehicular environment. The simulation results of this RLSHHOA confirmed improved mean channel utilization rate by $17.28 \%$, mean emergency message delivery rate by $18.32 \%$, mean neighborhood awareness rate by $19.82 \%$ and mean location rate by $23.21 \%$, better than the compared positioning schemes. However, the performance of this RLSHHOA was identified to still possess a room of improvement in neighborhood awareness rate and channel utilization rate on par with the compared approaches.

Furthermore, Raccoon Optimization Algorithm-based Localization Scheme (ROALS) was proposed for determining ranging measurements that aids in better estimation of NLOS node positions under warning message delivery [22]. This proposed ROALS was proposed with the guarantee of minimizing latency and maximized reliability during the exchange of position information during emergency situations. It adopted the food rummaging sytles of raccoons for speedening up the process of global and local search essential during the position determination of NLOS nodes. It facilitated better localization of NLOS nodes based on active learning that aided in better adaptability with maximized probability of determining distance information. It also utilized different distance information such as number of vehicles, distance information error and vehicle trajectory for attaining significant localizations. The simulation results of this ROALS scheme confirmed an improved mean channel utilization rate by $13.46 \%$, mean emergency message delivery rate by $14.92 \%$, mean neighborhood awareness rate by $17.38 \%$ and mean location rate by $21.84 \%$, better than the compared positioning schemes. An improved Grey Wolf optimization algorithm-based localization scheme (IGWOALS) was proposed for attaining integrity services and localization process that are essential for handling the issues in NLOS situations [23]. This IGWOALS adopted the hunting and encircling phenomenon of grey wolves into the search agents for the objective of identifying the position of NLOS nodes during emergency message dissemination. It was proposed with the merits of survival of fittest and biological evolution for establishing the compromise between exploitation and exploration. It included differential evolution for preventing the algorithm from being struck into the local point of optimality. It was confirmed to be significant in minimizing mean square error rate and latency with maximized warning message delivery rate and neighborhood awaremess rate. 
In addition, Grey Wolf and Crow Search Optimization Algorithm-based Localization Scheme (GWCSOALS) was proposed for predicting the location of unknown NLOS nodes in the vehicular network [24]. İt was proposed for the objective of minimizing the objective function formulated for the problem of NLOS localization process without being trapped into the local optimal poinnt. It handled the problem of broadcast storm and ensured relaibility during warning message delivery. This strategy included the merits of cooperative localization agent that derived the parameters associated with the geographical information and Time of Arrival (ToA). It was capable enough in positioning NLOS nodes through the intelligent strategy, which is analogous to the social attacking characteristics and conduit behavior of gray wolves and crows, respectively. It was also phenomenal in balancing the deviation between intensification and extensification such that location of NLOS nodes are determined with maximized accuracy. The simulation results of GWCSOALS also proved reduced localization error rate of $2.36 \%$ with mean neighborhood awareness rate of $12.38 \%$ and average warning message delivery rate of $11.82 \%$, superior to the compared approaches. An Integrated Seagull and Thermal Exchange Optimization Algorithm (ISTEOA)-based NLOS node positioning scheme was proposed for ensuring relaible warning message delivery [25]. This ISTEOA adopted three variants of optimization strategy based on the distance estimated between the unknown NLOS node and the reference nodes that could be possibly utilized for better localization. It handled the tradeoff between the exploitation and exploration over the search space for attaining potential localization of NLOS nodes. It adopted roulette wheel method for chosing an ideal optimization algorithm from the feasible three variants of optimization algorithm during the implementation of the algorithm in the search space. It finally combined the benefits of heat exchange formula into the seagull attack mode for enhancing the exploitation potentialities. The results of ISTEOA scheme proved better mean channel utilization rate by $11.24 \%$, mean emergency message delivery rate by $14.86 \%$ and mean neighborhood awareness rate by $13.64 \%$, compared to the existing positioning schemes.

\section{Proposed Spotted Hyena and Simulated Annealing Optimization Algorithm (SHSAOA)- based NLOS node positioning scheme}

This Spotted Hyena and Simulated Annealing Optimization Algorithm (SHSAOA)-based NLOS node positioning scheme is proposed with the merits of global optimality of SHOA and local optimality benefits of SA in order to establish the balance between exploration and exploitation in the search process. The SHOA algorithm is capable of determing the optimal or near optimal solution in the search space with respect to the fitness function. In the proposed SHSAOA scheme, SA algorithm is integrated into SHOA for two core reasons, such as i) enhancing the optimal solution identifed by SHOA at the end of every individual iteration and ii) improving the final solution determined by SHOA. This integration of SA with SHOA is considered to achieve better spatial search independent to the number of obstacles that contribute towards NLOS situations in the network. The detailed view of the proposed SHSAOA-based NLOS node positioning scheme is presented as follows.

\subsection{Primıtıve Spotted Hyena Optimization Algorithm (SHOA)}


Spotted Hyena Optimization Algorithm (SHOA) is proposed based on hunting charateristics of large carnivorous canine spotted hyena that generally hunts the medium and large sized herbivorous animals like impala, wildebeesta and zebras. This SHOA completely models the group hunting pattern exhibited by spotted hyenas during the process of encircling and attacking the prey. İt inherits the intellegent capability of spotted hyenas that aids in identifying their relative and other individuals based on multiple senses. İt included the capability of spotted hyenas that ranks the relationship of the same race and individuals with high potential are given more trust prioroty in the population. It also adopts the high sucess rate of spotted hyenas towards the process of group hunting phenomenon possessed by its nature. These merits of SHOA is utilized for determining the accurate location of NLOS during the process of emergency message dissemination.

\subsubsection{Process of prey encircling}

This phase of SHOA adopys the capability of intelligently knowing the location of the prey and encircle them. In this primitive SHOA, the reference nodes termed as the current best candidate solution is considered to be very close to the prey as the search space is not known in prior. The locations of other search agents are updated after the best search solution is defined and the mathematical model portraying this behavior is described by the following equations:

$$
\begin{gathered}
\overrightarrow{S H}(k)=\left|\overrightarrow{C_{V(A)}} * P V_{N L O S(x)}-P V_{\text {ref }(x)}\right| \\
\overrightarrow{p v(x+1)}=p v_{N L O S}(x)-C_{V(B)} S \overrightarrow{H(k)}
\end{gathered}
$$

Where, $S \vec{H}(k)$ represents the distance between the NLOS nodes and reference nodes at a current iteration $x . P V_{N L O S} \rightarrow(x)$ and $P V_{r e f}(x)$ is the position vectors of the NLOS nodes and reference nodes with the coefficient vectors $\overrightarrow{C_{V(A)}}$ and $\overrightarrow{C_{V(B)}}$ respectively. Further, the coefficient vectors are computed based on Equation (3), (4) and (5) respectively.

$$
\begin{aligned}
& C_{V(A)}=2 r_{v} \vec{d}_{s(1)} \\
& 2 \vec{g} r_{v} \overrightarrow{d_{s(2)}}-\vec{g} \\
& \vec{g}=5-\left(\text { Iter } *\left(\frac{5}{\text { Iter_Max }}\right)\right)
\end{aligned}
$$

$$
\overrightarrow{C_{V(B)}}=
$$

In this context, the value of ' $k$ ' linearly reduced from 5 to 0 for potential balancing between the rate of exploitation and exploration over the increasing number of iterations satisfying the condition $1 \leq$ Iter $\leq$ Iter_Max. This systematic decrease in the value of ' $k$ ' increases the degree of exploitation as the value of iteration increases. Moreover, are random vectors ranging between 0 and 1 . 


\subsubsection{Process of hunting NLOS nodes}

In general, spotted hyenas live and hunt in groups and completely depends on the trusted friends network (number of reference nodes) and its position in localizing the prey (NLOS nodes). In this SHOA approach, the best search agent is considered to possess the knowledge pertaining to the prey location (NLOS nodes) for defining the hunting behavior of spotted hyenas (search agents implemented over the vehicular reference nodes). The remaining search agents apart from the best search agent forms a cluster with trusted reference nodes that converges towards the best search agent with determined best solutions saved and updated positions identified so far based on Equation (6), (7) and (8), respectively.

$$
\begin{aligned}
& \overrightarrow{D_{g}}=\left|\overrightarrow{C_{V(A)}} \overrightarrow{P_{k(b)}}-\overrightarrow{P_{k(o-s)}}\right| \\
& \overrightarrow{P_{k(o-s)}}=\overrightarrow{P_{g}}-\overrightarrow{E_{P}} \overrightarrow{C_{V(B)}} \\
& \overrightarrow{C_{g}}=\overrightarrow{P_{k(o-s)}}+\underset{P_{k(o-s)+1}}{\vec{\longrightarrow}}+\ldots . .+P_{k(o-s)+N} \rightarrow
\end{aligned}
$$

Where, $P_{k(b)}$ and $P_{k(o-s)}$ represents the location of the best reference nodes and the location of the other reference nodes that aids in localizing the NLOS nodes in the network. Further, the number of spotted hyena search agent is determined based on Equation (9).

$S H_{S A(N)}=N o_{\text {Count }}\left(\overrightarrow{P_{g}}, \overrightarrow{P_{g+1}}, \overleftrightarrow{P_{g+2}}, \ldots \ldots, \overrightarrow{P_{g}}+\vec{Z}\right)$

Where, $\vec{Z}$ is a random vector that ranges between the value of 0.5 and $1 . N o_{\text {Count }}$ defines the possible number of solutions that includes the number of all candidate solutions after adding with the random vector $\vec{Z}$. This parameter $N o_{\text {Count }}$ is very similar to the best optimal solution in the space of search. Moreover, $\overrightarrow{C_{g}}$ is the total collection of $S H_{S A(N)}$ optimal solutions.

\subsubsection{Exploitation Phase (Prey attacking)}

This phase of exploitation (prey attacking phase of SHOA is modeled mathematically by decreasing the value of the vector $\vec{g}$. The deviation in the vector $\overrightarrow{E_{P}}$ is also minimized for dynamically changing the value of the vector $\vec{g}$, which need to be decreased from 5 to 0 over the iteration processes. When the value of $\left|E_{P}\right|<1$, then the exploitation process of attaining local optimization (spotted hyena search agents move towards the positioning of NLOS nodes in the neighborhood) is achieved. The mathematical formula considered for potraying the process of exploitation is presented in Equation (10)

$\vec{P}(x+1)=\frac{\overrightarrow{C_{g}}}{S H_{S A(N)}}$ 
Where, $\vec{P}(x+1)$ stores the best solution and updates the position of the remaining search agents based on the best search agents position. Thus, this SHOA permits the search agents to localize the position of the NLOS nodes in the network.

\subsubsection{Exploration Phase (Prey searching)}

In the phase of exploration, the prey is searched by the spotted hyena search agent based on their individual position that resides inside the vector $\overrightarrow{C_{g}}$. These search agents move away from one another depending on the distance between the reference nodes and the unknown search agents during the process of searching the prey (NLOS nodes). This phase also utilizes the vector $\overrightarrow{E_{P}}$ with random values that ranges between -1 and +1 for enforcing the search agents to approach very closer to the unknown NLOS nodes. This use of vector $\overrightarrow{E_{P}}$ with random values aids the SHOA algorithm to perform global optimization in the search space. In specific, the value of $\left|E_{P}\right|>1$ facilitates the search agent to move away from the NLOS nodes. Thus, reference nodes are used for positioning the NLOS nodes in the vehicular network. Further, the exploitation is also improved by another vector $\overrightarrow{C_{V(A)}}$. This vector $\overrightarrow{C_{V(A)}}$ presented in Equation (3) possesses random values in order to provide random weights during the prediction of NLOS nodes' position. Furthermore, the vector condition $\overrightarrow{C_{V(A)}}>1$ can be considered instead of $\overrightarrow{C_{V(A)}}<1$ for incorporating random behavior into SHOA algorithm that targets on maximized diversity in the search space. This use for vector $\overrightarrow{C_{V(A)}}$ thereby helps in preventing local optimal point with maximized exploration (global optimization process). This SHOA algorithm gets terminated after the satisfaction of the termination criteria. The possible capabities of this SHOA algorithm is listed as follows.

i) It is capable of storing best solutions (location of NLOS nodes predicted or localized directly or through reference nodes) and rembering them over the entire iteration course.

ii) It used the random vector of $C_{V(A)}$ and $\overrightarrow{E_{P}}$ for supporting the candidate solution to possess hyper-sphers with various random positions necessary during exploitation and exploration process.

iii) It included a encircling mechanism that defines a neighborhood circular-shaped region around the solutions with the possibility of being extended as a hyper-sphere search space.

iv) It adjusts the value associated with the random vector $C_{V(A)}$ and $\overrightarrow{E_{P}}$ for potential swirching between exploration and exploitation.

v) It inherited a hunting methodology that permits candidate solution to position the feasible location of the unknown NLOS nodes in the network.

However, this SHOA algorithm is better in global optimization (exploration), but still possesses a room of improvement in the local optimization process (exploitation). Hence, local 
optimizing intelligent metaheuritic improved simulated annealing (SA) (presented in section 3.2) is integrated into SHOA for establishing better balance between the process of exploitation and exploration in the search space.

\subsection{Improved Simulated Annealing}

The traditional Simulated Annealing (SA) algorithm was proposed by Kirkpatrick et al. [26] as a single solution-based meta-heuristic optimization algorithm that inspires the annealing process of metal working. The procedure of annealing refers to the optimal arrangement of molecues associated with metal particles that concentrates on the minimization of mass potential energy followed by gradual cooling of molecules after subjected to maximized heating process. This SA algorithm adopts a parameter of variable temperature for facilitating iterative movement in order to mimick the metal annealing transactions. In SA algorithm, the objective function value represent the energy of the thermodynamic system which needs to be explored by the search agents for updating their position through random walk at each and every iteration. This random walk strategy is applied by estimating the deviation between the change in temperature identified during the anneal process. This classical SA allows large movements at high temperatures in the search space and accepts only the solutions whose probability does not get improved compared to the existing optimal value. Thereby, SA prevents getting stuck into a local point of optimality. In general, SA starts with an initial solution $S_{\text {Initial }}$ and a mechanism of cooling $C_{M}$ with each element temperature $t_{(i)}$ with respect to individual iteration ' $i$ 'is specified in Equation (11)

$C_{M(i)}=\left\{t_{(1)}, t_{(2)}, \ldots \ldots, t_{(n)}\right\}$

Then, neighboring solution $\left(N_{S o l(i)}\right)$ close to the current solution $\left(C_{S o l(i)}\right)$ at the end of every individual iteration is generated using random perturbation. Further, temperature $t_{(i)}$ impacts the strength of perturbation. When the temperature is low, small pertubations are visualized. In contraction, bigger perturbation is identified with high temperature. Further, the solutions $\left(N_{\operatorname{Sol}(i)}\right)$ and $\left(C_{S o l(i)}\right)$ are compared for identifying the best solution. The preservation of the current best solution $\left(C_{S o l(i)}\right)$ or replacing the current solution with newly estimated neighboring solution $\left(N_{\text {Sol }(i)}\right)$ completely depends on the probability $P_{\text {accept }}$. This probability of acceptance (formulated based on minimization function) considered for accepting a solution is determined based on Equation (12)

$P_{\text {accept }}=\left\{\begin{array}{cl}e^{-\left(\frac{f\left(N_{\text {Sol }(i)}\right)-f\left(C_{\text {Sol }(i)}\right)}{t_{(i)}}\right)} & \text { If } f\left(N_{\text {Sol }(i)}\right)-f\left(C_{\text {Sol }(i)}\right)>0 \\ 1 & \text { If } f\left(N_{\text {Sol }(I)}\right)-f\left(C_{\text {Sol }(i)}\right) \leq 0\end{array}\right.$

In this case, the temperature is decreased based on the employed cooling mechanism without considering whether the new solution was accepted or rejected. This iterative process is continued unless the temperature reaches the defined final value. However, the exploration and exploitation capabilities still possesses a room for improvement. 
In this proposed scheme, an improved simulated annealing algorithm using the trajectorybased principles of generation and reannealing (GR-ISA) is utilized for enhancing the rate of convergence towards the superior optimal front. In specific, this enhancement in convergence rate attained by the improved SA algorithm exhibits better uniformity and diversity in the solutions distribution. In the first phase, GR-ISA used diversfied generation strategies for suitable definition of candidate solutions at diversfied phases of the search process. Then, a reannealing process is applied in the second phase at a very low temperature for search intensification based on the current solutions determined in the first phase. This GR-ISA is considered to offer good results in improving the exploitation degree to the maximized level compared to the other local optimizing algorithms contributed to the literature. It included the benefits of archive to store non-dominated solutions identified until the current iteration through the concept of domination amount. In general, the domination amount archive is utilized for systematically storing the non-dominating solutions that are determined during the optimization process. When a new dominanting solution is identified, then the non-dominating solutions are discarded depending its potentialities. Moreover, the size of the archive used in this GR-ISA is considered be highly dynamic. But, GRISA is improved with a new archival mechanism through which none of the non-dominating solutions determined until the current iteration gets discared during the process of evaluation. In this context, the domination degree that could be possibly calculated between any two generated solutions $u_{1}$ and $u_{2}$ is depicted in Equation (13)

$\operatorname{Dom}_{\text {Degree }}\left(u_{1}, u_{2}\right)=\prod_{i=1, f i t}^{N}\left(u_{1}\right) \neq f i t\left(u_{2}\right) \mid \frac{f i t\left(u_{1}\right)-f i t\left(u_{2}\right)}{f^{i t_{\text {Max }}-f i t_{\text {Min }}} \mid}$

Where, fit $\left(u_{1}\right)$ and $f i t\left(u_{2}\right)$ refers to the fitness function value determined for two generated solutions $u_{1}$ and $u_{2}$ based on $f i t_{M a x}$ and $f i t_{M i n}$ as the maximum and minimum threshold of fitness that could be possibly determined during the optimization process of NLOS localization. This aforementioned domination concept equation is vital in preventing the issues that are more common during the strict utilization of Pareto dominance. Further, two important improvement such as adaptive generation process and reannealing are included in the utilized GRISA algorithm on par with the traditional SA algorithm. This inclusion of improving strategies concentrates on the objective of enhancing diversity rate and handles the issues of convergence during optimization process.

In GR-ISA algorithm, a potential generation procedure is included for generating candidate solutions by deriving the merits of the trajectory-based charateristics of SA throughout the algorithmic development process. This significant generation process targets on identifying particular solutions among the non-dominating solutions determined until the current iteration to explore new ways for constructing candidate solutions. The new generation strategy aids at finding the best feasible non-dominating solution sets associated with the Pareto front. It is implemented for preventing the candidate solution generation in the neighborhood of the current solution, since it is inefficient in finding non-dominating solutions in the different regions of the search space. On the other hand, Reannealing is used for improving the non-dominated solutions identified in the 
previous generation phase for increasing the selection pressure towards the Pareto front. This reannealing process used in GR-ISA algorithm utilizes the archival solutions for attaining the best optimal solutions by focussing on the search process that specifically targets on the previously defined Pareto front.

\subsubsection{Adoption of novel solution generation process}

The process of implementing GR-ISA algorithm starts with the definition of an initial solution $\left(U_{\text {Initial }}\right)$ identified from the search space and setting the current solution $\left(U_{\text {Current }}\right)$ equal to $U_{\text {Initial }}$ with a defined initial temperature $\left(T_{\text {Init }}\right)$. In specific, the parameter $T_{\text {Init }}$ is computed based on the parameter of elasticity of acceptance. Then, the process of iteration gets initaied by the generation of candidate solutions $\left(U_{\text {Cand }}\right)$. Once the selection of new candidate solutions $\left(U_{\text {Cand }}\right)$ are selected with constraints verification, then the process of evaluating the objective functions continues until the termination criteria and maximum number of iterations considered for implementation is met. Further, the candidate solution is accepted or rejected based on the Metropolis criterion depending on the status of dominance identified based on the comparison achieved between $U_{\text {Cand }}$ and $U_{\text {Current }}$ solutions from the non-dominated set stored in an archive ( $U_{\text {Archive }}$ ). If the candidate solution $U_{\text {Cand }}$ is better than the $U_{\text {Current }}$ solutions, then the $U_{\text {Cand }}$ is considered as th new $U_{\text {Current }}$ solution. This process of generation is iterated until an equilibrium is achived and the reduction in the temperature parameter is visualized. Furthermore, the temperature of the sucessive annealing step $T_{N e w}$ is calculated based on the geometric annealing schedule by multiplying the temperature of the current iteration $T_{\text {Current }}$ with the factor of cooling $\alpha$ (generally lies between 0.8 and 0.99 ) for the reducing the temperature as depicted in Equation (14).

$T_{\text {New }}=\alpha \times T_{\text {Current }}$

The main advantage of geometric annealing schedule lies in its characteristics that exhibit better balance between the exploitation and exploration in the solution space. This process of generation continues to target on the temperature reduction until the termination criterion is satisfied. Finally, the complete set of non-dominating solutions determined until the satisfication of termination criteria is stored in the archive. In this phase, the generation of new candidate solution utilizes the archive for guaranteeing search relocation in the search phase. In specific, four core generation processes such as GP-I, GP-II, GP-III and GP-IV are used for generating the candidate solutions depending on the different thresholds of temperature. These different temperature thresholds plays an anchor role in selecting the generation process with computed probabilties in order to control the entire optimization process. This potential capability inherent with the probability of selecting generation process focus on generating new solution that helps in facilitating better global search initially and ensure more localized search at the end of the optimization. The generation processes of GP-I, GP-II, GP-III and GP-IV used in the proposed GR-ISA algorithm is presented as follows. 
GP-I: This process uses current solution for generating the candidate solution.

GP-II: It selects a random solution from the archive for generating the candidate solution.

GP-III: It choses specific solutions from the archive that are associated with specific portions of the Pareto front during the generation of candidate solutions.

GP-IV: It selects solution present in the knee region of the archive for generating candidate solutions.

The above mentioned candidate solution generating process is selected based on two probabilities $\left(P_{\text {Select (1) }}, P_{\text {Select (2) }}\right)$ and with three temperature thresholds $\left(T_{T h 1}, T_{T h 2}, T_{T h 3}\right)$. This process of generation also depends on limit of the number of iterations $\left(\right.$ Iter $\left._{\text {Min }}\right)$ associated with the execution of GP-IV. Three temperature thresholds controls the entire new generation process, once it is induced in the optimization process. Moreover, these temperature thresholds satisfies the condition $T_{T h 1}>T_{T h 2}>T_{T h 3}$. The generation process GP-I and GP-II is initiated only when the temperature of the current iteration mets the condition $T^{\text {Current }}>T_{T h 1}$ and $T^{\text {Current }}<T_{T h 2}$, respectively. Further, the generation process GP-III and GP-IV is included for implementation, only when the temperature of the current iteration satisfies the criterion $T^{\text {Current }}<T_{T h 2}$ and $T^{\text {Current }}<T_{T h 3}$, respectively. The selection probabilities $P_{\text {Select(1) }}$ and $P_{\text {Select(2) }}$ are also calculated in this situation for introducing the generating process during implementation. When the temperature is lower than $T_{T h 1}$, then the possibility of selecting more than one generation process arises. Thus, a random number $\operatorname{Rand}_{V(1)}$ is used based on uniform distribution for determining the probability used for selecting GP-I and GP-II with equalized priority. The value of random number $\operatorname{Rand}_{V(1)}$ calculated for inducing the selection of GP-I and GP-II processes are presented in Equation (15) and (16)

$\operatorname{Rand}_{V(1)}=1-2 \times P_{\text {Select }(1)}$

$\operatorname{Rand}_{V(1)}=2 \times P_{\text {Select }(1)}$

In this case, the value of $P_{\text {Select(1) }}$ ranges between 0 and 0.5 , which is selected depending on the importance provisioned to GP-I and GP-II processes.

When the temperature is lower than $T_{T h 2}$, then a random number $\operatorname{Rand}_{V(2)}$ that follows uniform distribution is utilizing determining the probability that is used for selecting GP-II or GPIII process. The value of random number $\operatorname{Rand}_{V(2)}$ calculated for selecting GP-II or GP-III is portrayes based on (17)

$\operatorname{Rand}_{V(2)}=P_{\text {Select(2) }}-\left(\frac{T^{\text {Current }}}{T_{\text {Init }}}\right) \times P_{\text {Select(2) }}$ 
Where, the functional probability of $P_{\text {Select(2) }}$ varies depending on the temperature rate determined based on $T^{\text {Current }}$ and $T_{\text {Init }}$. Moreover, this probability of $P_{\text {Select(2) }}$ with highest value of $T^{\text {Current }}$ concentrates on minimum selection of GP-III and maximum selection of GP-II processes. On the other hand, $P_{\text {Select(2) }}$ with $T^{\text {Current }}$ approaching targets on minimum selection of GP-II and maximum selection of GP-III processes. The possibility of selecting GP-II and GPIII complete depends on the fall in temperature. This process of selecting GP-II in the first temperature threshold ensures more complete search in the earlier stage of optimization. GP-III offers more localized optimization search in the last iterations around the uncrowded, middle and extreme regions of the search space.

GP-IV is finally selected with $P_{\text {Select(1) }}$ probability, when the temperature is lower than $T_{T h 3}$. The number of interactions between candidate solutions is considered to be minimized with temperature is estimated to be less than $T_{T h 3}$. Further, Iter ${ }_{\text {Min }}$ is used for search intensification during the selection of process GP-IV. A counter termed $C n t_{\text {Iter }}$ is initially set to zero during GPIV selection, until $C n t_{I t e r}$ is greater or equal to Iter ${ }_{\text {Min }}$. This counter $C n t_{I t e r}$ is monotonically increased during the selection of GP-I for a number of iterations, such that more localized search is introduced using the knee portion of Pareto front until the criterion $\left(\mathrm{Cnt}_{\text {Iter }} \geq\right.$ Iter $\left._{\text {Min }}\right)$ is satisfied. Therefore, potential candidate solutions are identified during the employment of novel solution generation process that are considered as input to the subsequent reannealing process.

\subsubsection{The process of reannealing}

In this second phase of GR-ISA algorithm, a reannealing temperature $\left(T_{\text {Reaneal }}\right)$ is defined based on the two temperature levels estimated before the end of the previous novel solution generation process. This parameter of $T_{\text {Reaneal }}$ is a low temperature value that aids in promoting a more localized search related to a minimized probability of accepting candidate solutions with large deteriorations in the objectives. The non-dominating solutions determined in the first phase of annealing is partitioned into different collections depending on the objectives' range. Then, each of the partition is promoted with the objective of improving the feasible non-dominating solutions determined in the previous phase for identifying a better pareto front. Ar this juncture, the nondominated solutions are chosen as the pareto front thresholds plus one solution considered from each individual partitioned group. This process of reannealing considered in GR-ISA is a local searching process, which is imposed around the intial solutions determined during the previous phase. This reannealing process is applied after the completion of process GP-I for generating new candidate solutons that aims at localized and intensified search around the parts of the Pareto front. The number of partitions or groups considered in the annealing process is proportional to the number of candidate solutions determined in the previous phase. In this GR-ISA algorithm, the number of partitions is computed based on Equation (18).

Partition $_{\text {Cnt }}=\frac{U_{\text {Solution-Population }}}{U_{\text {Solution-potential }}}$ 
Where, $U_{\text {Solution_Population }}$ and $U_{\text {Solution_Potential }}$ represents the number of solutions present in the population and the number of solutions that potentially satisfy the termination criteria of the utilized fitness function. Hiwever, higher number of partitions aids in attaining better final pareto fronts as additional reannealing strategies are induced in every individual Pareto front partitions. This GR-ISA algorithm is thereby determined to be potential in attaining indespensable local optimization, such that its inclusion into SHOA algorithm plays a remarkable role in maintaining the balance between exploitation and exploration during NLOS node localization process.

\subsection{Process of Integrating SHOA and GR-ISA Algorithm}

This integration of SHOA and GR-ISA algorithm aids in determining the better solution (predicted locations of NLOS nodes) and high spatial search. This hybrid approach combines the merits of development-oriented search algorithm with the exploration-based population algorithm. Hence, this proposed SHSAOA model targets in improving the algorithmic performance in two dimensions such as, i) considering the local search as the global search operator during the process of searching in improving the prediction accuracy and ii) the primary algorithm carries out of the search and the secondary algorithm aids in strengthening the solution already determined by the initial algorithm. The primary SHOA algorithm is determined to exhibit better performance on par with most of the optimization algorithms but there is still a scope of improvement in its local search process. On the other hand, GR-ISA algorithm is phenomenal in imposing strong local search with the tendencies to enhance the local optimal solution during the hybridization process. It is proposed as a wrapper selection mechanism for integrating SHOA and GR-ISA algorithms. In specific, GRISA algorithm is included into SHOA for improving the utilization rate to the maximized degree. In the first hybridization process, GR-ISA algorithm is only employed as a portion of SHOA algorithm, and GR-ISA and SHOA algorithms are executed once for every individual iteration. In the second hybridization process, initially SHOA algorithm is used for searching the optimal solution, which further included GR-ISA for searching the current optimal solution. In addition, Figure 1 presents the flowchart of the proposed SHSAOA positioning algorithm that integrates the merits of SHOA and GR-ISA algorithm for NLOS localization process. 


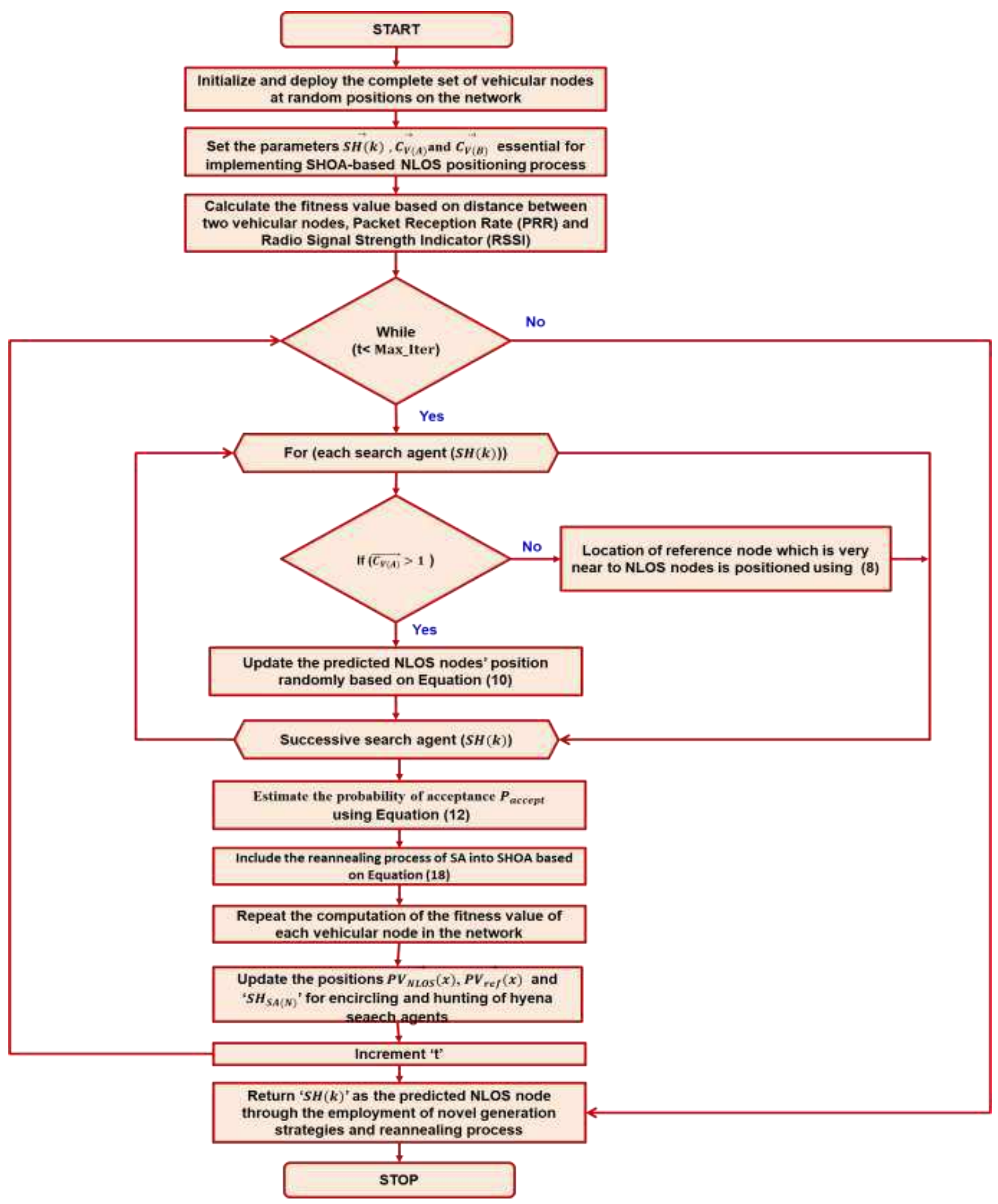

Figure 1: Flowchart of the proposed SHSAOA-based NLOS positioning algorithm 
İnt1ally, the complete set of vehicular nodes are deployed in the entire network area among which search agents are randomly selected among them. Then, the parameters associated with the

search agents $\overrightarrow{S H}(k)$ and its behavioral characteristic cofficients $\overrightarrow{C_{V(A)}}$ and $\overrightarrow{C_{V(B)}}$ essential for implementing SHOA-based NLOS positioning is set. Once the parameters are set, the fitness values used for predicting the location of NLOS nodes is determined based on distance between two vehicular nodes, Packet Reception Rate (PRR) and Radio Signal Strength Indicator (RSSI). This process of predicting NLOS nodes is carried until the maximum number of iterations for each search agent $\overrightarrow{S H}(k)$. When the behavioral coefficient is $\left(\overrightarrow{\boldsymbol{C}_{V(\boldsymbol{A})}}>\mathbf{1}\right)$, then the location of reference node which is very near to NLOS nodes is positioned using (8), Otherwise, the position of the NLOS nodes are randomly predicted based on Equation (10). However, the local optimization needs improvement and hence the GR-ISA algorithm is employed for estimating the acceptance probability $P_{\text {accept }}$ based on Equation (12). Further, the local optimization process is subsequently improved by including the reannealing process of GR-ISA into SHOA based on Equation (18). Furthermore, the positions $P V_{N L O S}(x), P V_{\text {ref }}(x)$ and ' $S H_{S A(N)}$ ' are subsequently used for attaining the encircling and hunting characteristics of hyena seaech agents. Finally, ' $S H(k)$ ' is returned as the predicted NLOS node through the employment of novel generation strategies and reannealing process

\section{Simulation Results and Discussion}

The simulation experiments of the proposed SHSAOA and the competitive NLOS localization schemes such as ROALS [22], GWCSOALS [24] and ISTEOA [25] are conducted using Veins version 4.5 framework which is integrated with SUMO and OMNET++ simulator. In specific, this SUMO simulator version of mobility 0.29 and network simulator version 5.0 of OMNET++ are integrated with the Veins framework. The utilization of SUMO and OMNET++ simulators helps in better definition of all the mobility parameters associated with the vehicle and the comprehensive parameters of the vehicular network that follows the standard of IEEE 802.11p standard. This simulation environment used for implementation utilized the merits of $\operatorname{Krau} \beta$ model, which is configured with driver imperfection rate of 50\%. In other words, the vehicles possesses diversified behaviors with respect to lanes selection for driving in, acceleration and deceleration. The vehicles were set up with minimum and maximum speed values of $40 \mathrm{~m} / \mathrm{sec}$ and $80 \mathrm{~m} / \mathrm{sec}$, respectively. The communication range and power transmission of the vehicles during interaction is set to 300 meters and $20 \mathrm{~mW}$, respectively. The vehicular nodes are considered to disseminate beacons in the network with the frequency of $10 \mathrm{~Hz}$ and the complete beacons packets are transmitted through the control channel at the rate of 6 Mbits per second. In specific, the path loss model of log normal shadowing is utilized with the limit of sensitivity $-84.39 \mathrm{dBm}$. The beacons with RSSI values greater then the considered limit of sensitivity is only processed. The experimental results are completely determined based on 40 number of simulation that follows student $\mathrm{t}$-test distribution with a confidence interval of $95 \%$. In addition, Table 1 presents the comprehesnove view of the simulation parameters considered in the implementation of the proposed SHSAOA and the competitive NLOS localization schemes. 
Table 1: Simulation parameters used for implementing SHSAOA scheme

\begin{tabular}{|l|l|}
\hline \multicolumn{1}{|c|}{ Simulation Parameters } & \multicolumn{1}{c|}{ Values Used } \\
\hline Pattern of communication & V2V \\
\hline WIFI Technology & WAVE IEEE $802.11 \mathrm{p}$ \\
\hline Frequency of WIFI Technology & $5.89 \mathrm{GHz}$ \\
\hline Number of vehicles & 300 \\
\hline Rate of transmission & $6 \mathrm{Mbits}$ per second \\
\hline Thredhold of sensitivity & $-84.39 \mathrm{dBM}$ \\
\hline Transmission Frequency & $10 \mathrm{~Hz}$ \\
\hline Total number of vehicles & 500 \\
\hline Imperfection & 0.5 \\
\hline GPS frequency & $10 \mathrm{~Hz}$ \\
\hline Mobility Pattern & $\mathrm{Krau} \beta$ model \\
\hline Acceleration & $1.9 \mathrm{~m} / \mathrm{sec}^{2}$ \\
\hline Path loss model & Log Normal Shadowing \\
\hline Range of Communication & $300 \mathrm{~meters}$ \\
\hline Size of the emergency packet & $512 \mathrm{bytes}$ \\
\hline Wave Channel & Center Channel $(\mathrm{CCH})$ \\
\hline Transmission Potency & $20 \mathrm{~mW}$ \\
\hline Minimum and maximum speed of vehicles & $40 \mathrm{~m} / \mathrm{sec}$ and $80 \mathrm{~m} / \mathrm{sec}$ \\
\hline Warning message packet size & $512 \mathrm{bytes}$ \\
\hline
\end{tabular}

The proposed SHSAOA and the competitive ROALS, GWCSOALS and ISTEOA localization approaches are evaluated based on warning message delivery rate, neighborhood awareness degree, channel utilization degree, time incurred in NLOS node localization, and Localization error by varying the number of vehicular nodes, NLOS nodes and reference nodes in the network.

\subsection{Performance Evaluation of proposed SHSAOA with different vehicular nodes}

In this performance evaluation, the proposed SHSAOA and the competitive ROALS, GWCSOALS and ISTEOA localization approaches are compared based on warning message delivery rate, neighborhood awareness degree, channel utilization degree and time incurred in NLOS node localization with different vehicular nodes. Figure 2 and 3 depicts the warning message delivery rate and neighborhood awareness degree attained by the proposed SHSAOA over the competitive ROALS, GWCSOALS and ISTEOA localization approaches with different vehicular nodes. The warning message delivery rate and the channel utilization rate of the proposed SHSAOA mechanism is determined to be comparatively better than the competitive approaches independent to the scalable increase in the number of vehicular nodes. This improvement in warning message delivery rate is mainly due to the local optimizing capability of GR-ISA integrated into SHOA for achieving in better search efficiency that aided in rapid and relaible positioning of NLOS nodes that in turn enhanced the warning message delivery rate in the network. 
On the other hand, neighborhood awareness degree is also improved due to the tendencies of novel generation and reannealing approaches into SHOA during positioning process. Hence, the warning message delivery rate of the proposed SHSAOA scheme with different vehicular nodes is identified to be improved by $9.21 \%, 10.94 \%$ and $11.86 \%$, excellent over the competitive ROALS, GWCSOALS and ISTEOA localization approaches. The neighborhood awareness degree achieved by the proposed SHSAOA scheme with different vehicular nodes also is also confirmed to be improved by $8.92 \%, 9.76 \%$ and $10.44 \%$, significant to the competitive ROALS, GWCSOALS and ISTEOA localization approaches.

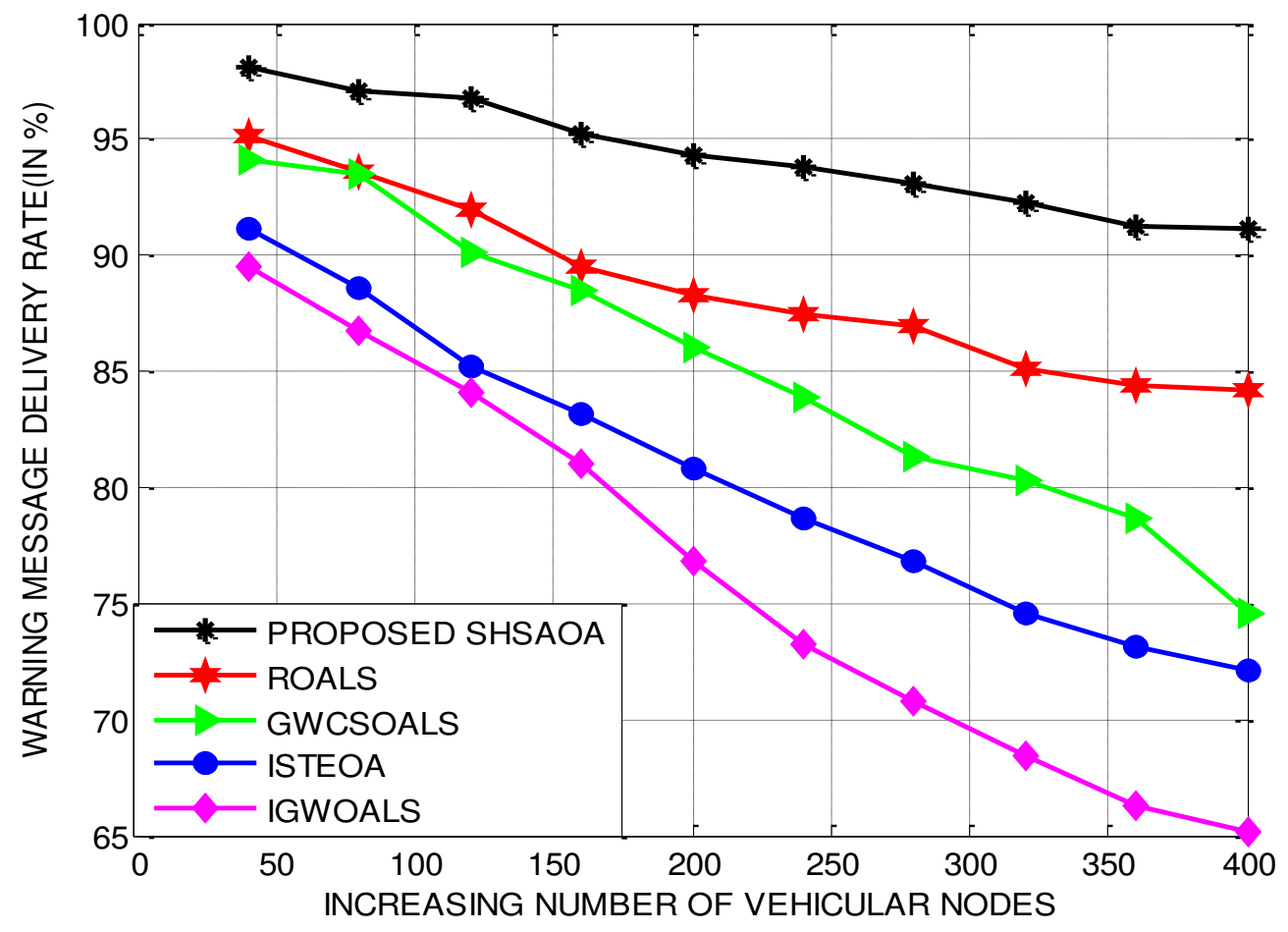

Figure 2: Proposed SHSAOA: Warning message Delivery Rate with different vehicles 


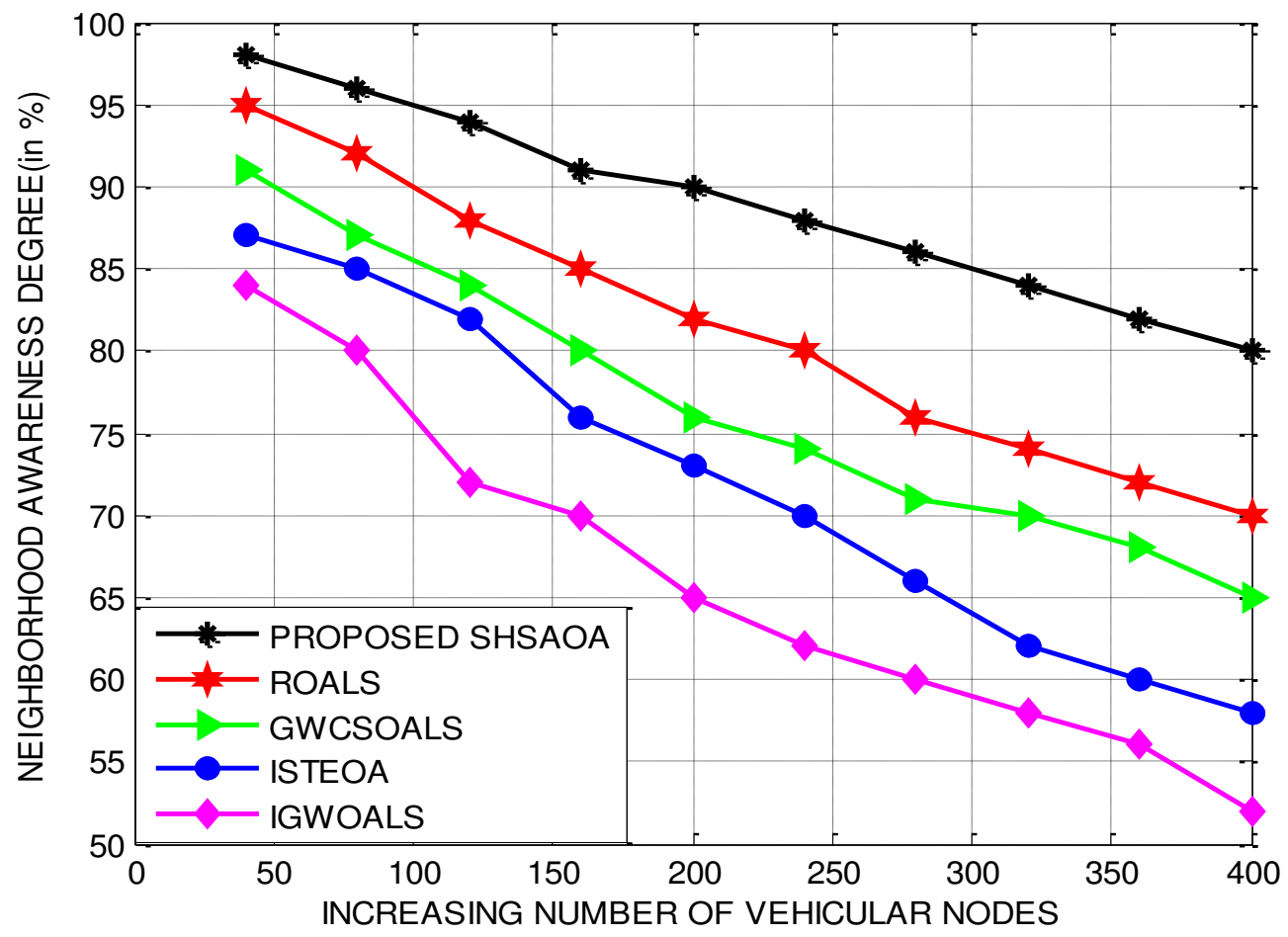

Figure 3: Proposed SHSAOA: Neighborhood Awareness Rate with different vehicles

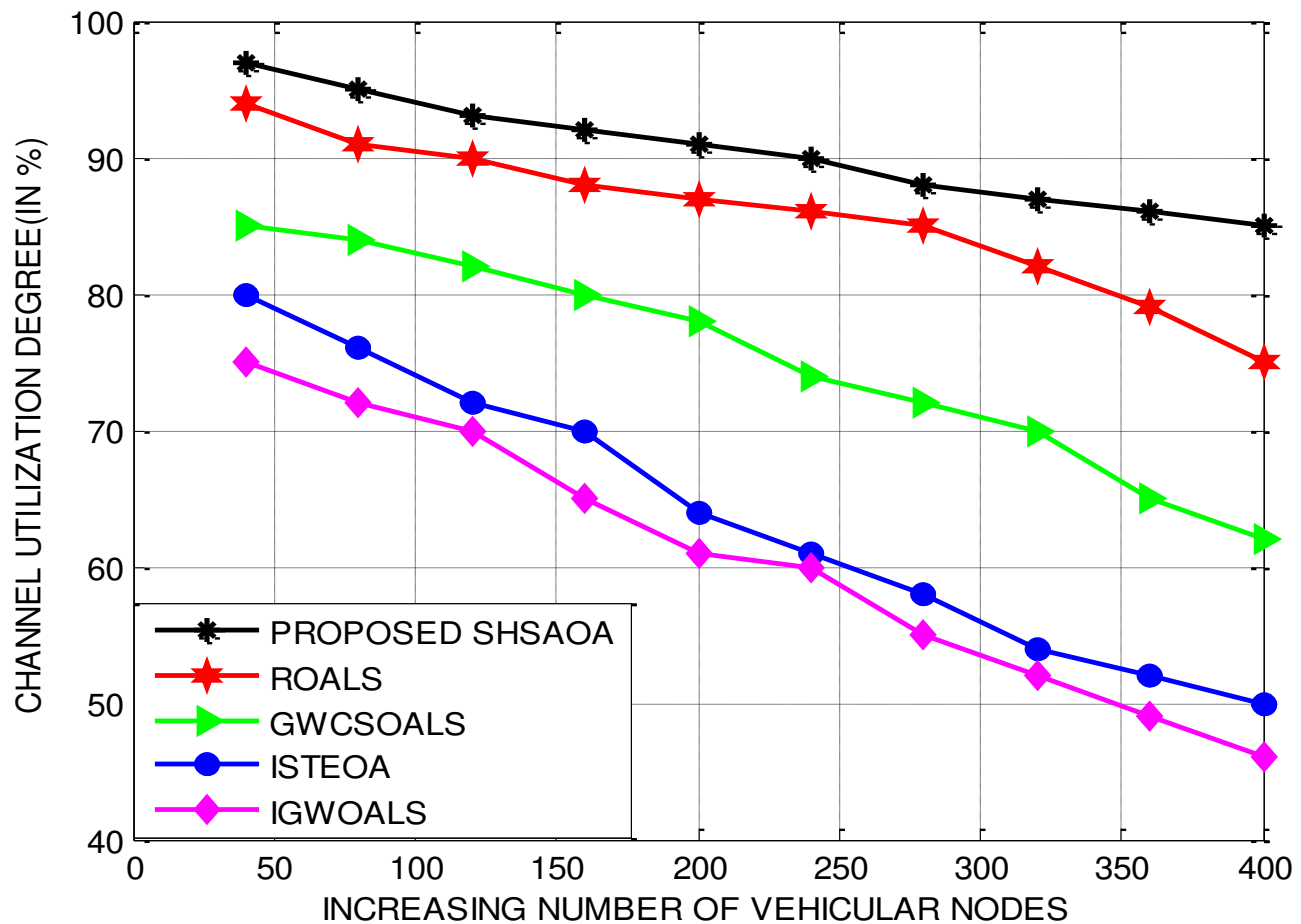

Figure 4: Proposed SHSAOA: Chanel Utilization Degree with different vehicles 


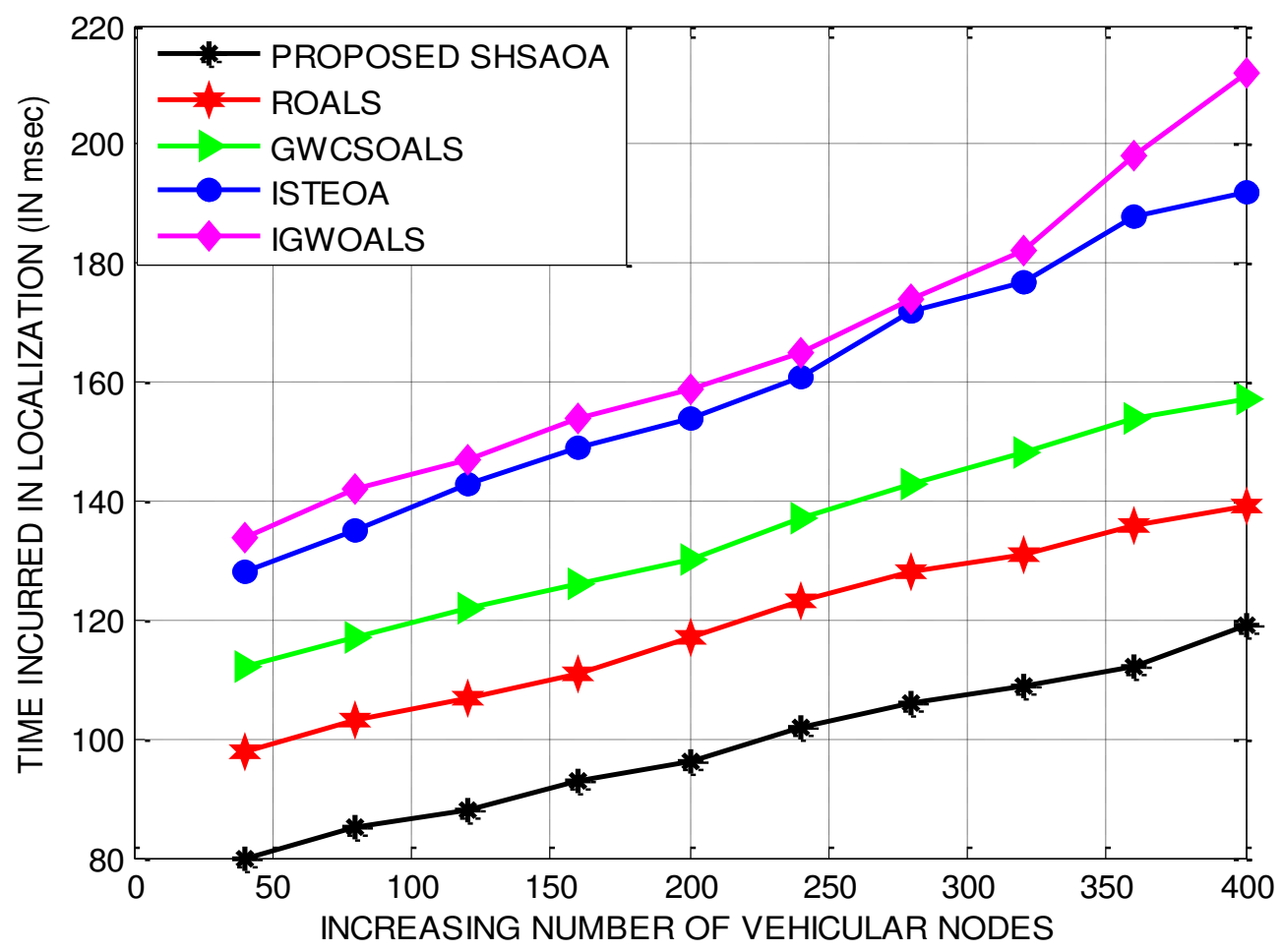

Figure 5: Proposed SHSAOA: Time incurred in localization with different vehicles

Figure 4 and 5 demonstrates the channel utilization degree and time in NLOS node localization incurred by the proposed SHSAOA over the competitive ROALS, GWCSOALS and ISTEOA localization approaches with different vehicular nodes. The channel utilization rate is significantly better than the competitive approaches, since the channel is used in a more adaptive manner through the localization potential of search equations included into SHOA. Hence, the channel utilization rate of the proposed SHSAOA scheme with different vehicular nodes also is also confirmed to be improved by $8.92 \%, 9.76 \%$ and $10.44 \%$, significant to the competitive ROALS, GWCSOALS and ISTEOA localization approaches. The time in NLOS node localization incurred by the proposed SHSAOA is minimized on par with the competitive approaches, since it has the capability of predicting the position of unknown nodes with reduced number of search agents. Moreover, the time in NLOS node localization incurred by the proposed SHSAOA scheme with different vehicular nodes also is also identified to be potentially minimized by $7.98 \%, 8.76 \%$ and $9.54 \%$, superior to the competitive ROALS, GWCSOALS and ISTEOA localization approaches.

\subsection{Performance Evaluation of proposed SHSAOA with different NLOS nodes}

In this performance evaluation, the proposed SHSAOA and the competitive ROALS, GWCSOALS, ISTEOA and IGWOALS localization approaches are compared based on localization accuracy, latency in warning message delivery rate, energy consumptions and number of warning messages lost with different NLOS nodes. Figure 6 and 7 depicts the localization accuracy and 
latency in warning message delivery achieved by the proposed SHSAOA compared to the baseline ROALS, GWCSOALS, ISTEOA and IGWOALS localization approaches with different vehicular nodes. The localization accuracy of the proposed SHSAOA is comparatively improved as the prediction is updated in each iteration based on multiple dimensions that attributed during better positioning. Moreover, latency involved in warning message delivery is also potentially minimized as it handled the impacts of obstacles independent to their shapes and sizes in the network. Hence, the localization accuracy of the proposed SHSAOA scheme with different NLOS nodes is identified to be improved by $9.21 \%, 10.94 \%$ and $11.86 \%$, excellent over the competitive ROALS, GWCSOALS and ISTEOA localization approaches. The latency in warning message delivery determined by the proposed SHSAOA scheme with different NLOS nodes also is also confirmed to be improved by $8.92 \%, 9.76 \%$ and $10.44 \%$, significant to the competitive ROALS, GWCSOALS, ISTEOA and IGWOALS localization approaches.

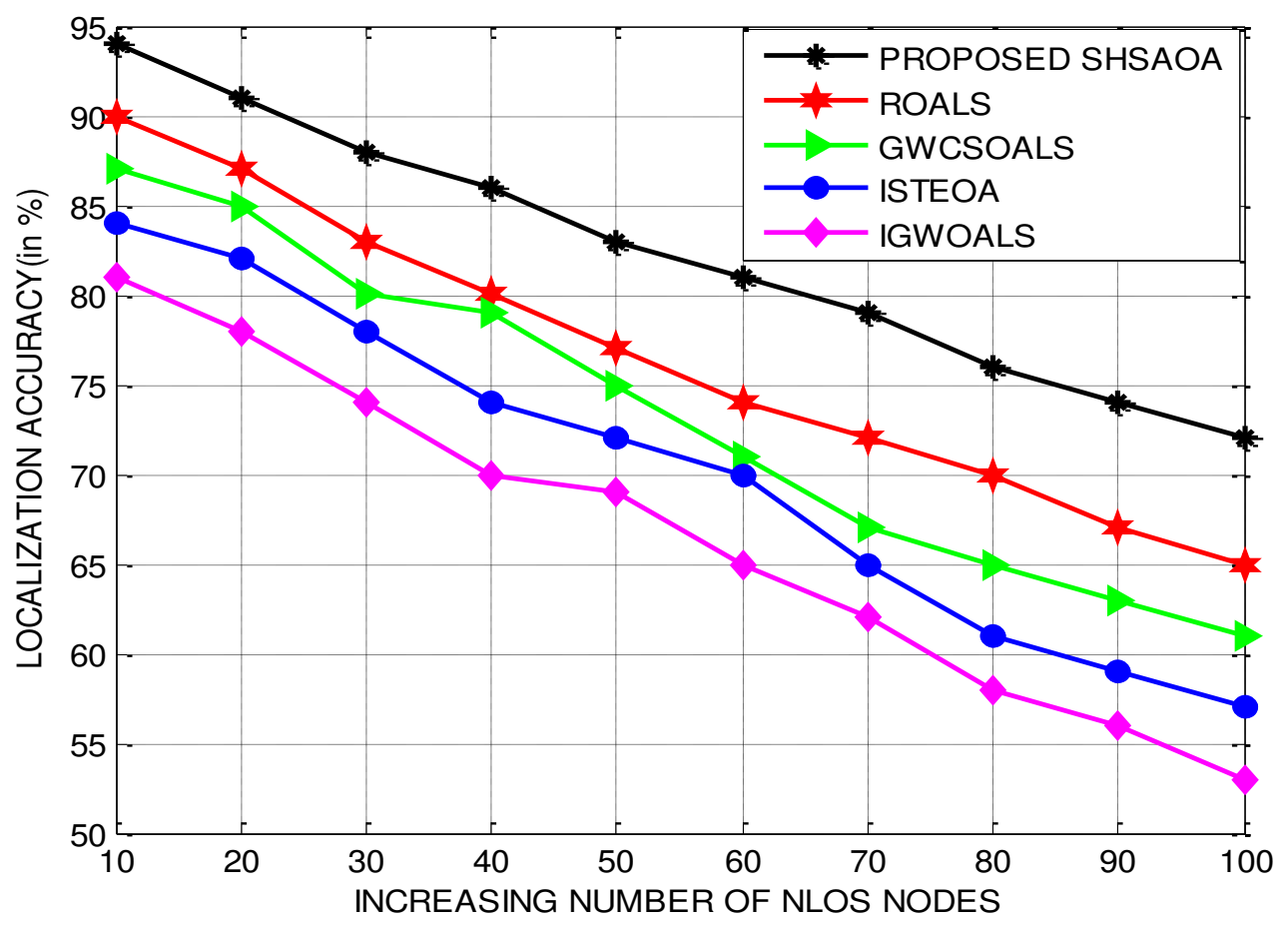

Figure 6: Proposed SHSAOA: Localization Accuracy with different NLOS vehicles 


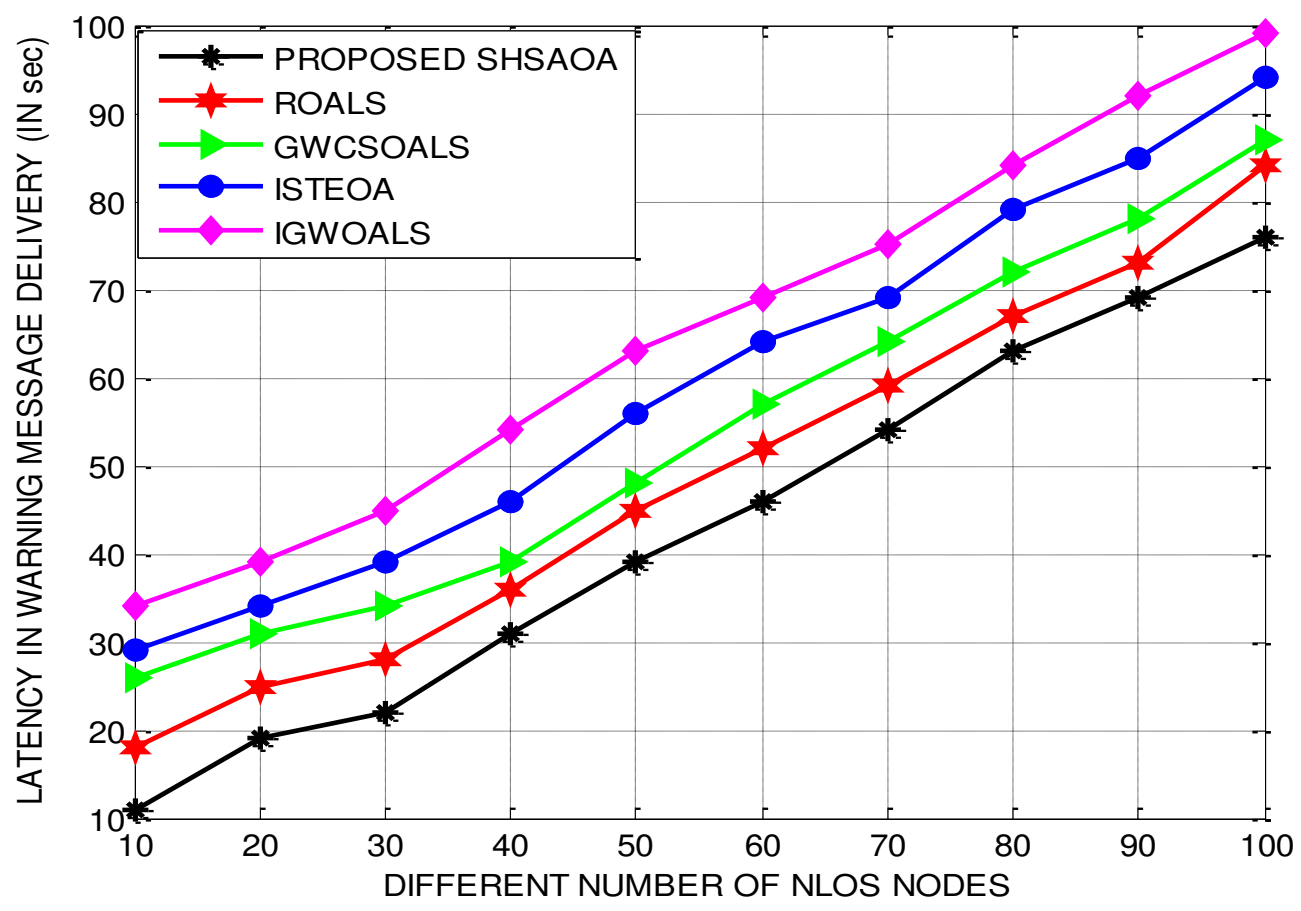

Figure 7: Proposed SHSAOA: Latency incurred during warning meassge delivery with different NLOS vehicles

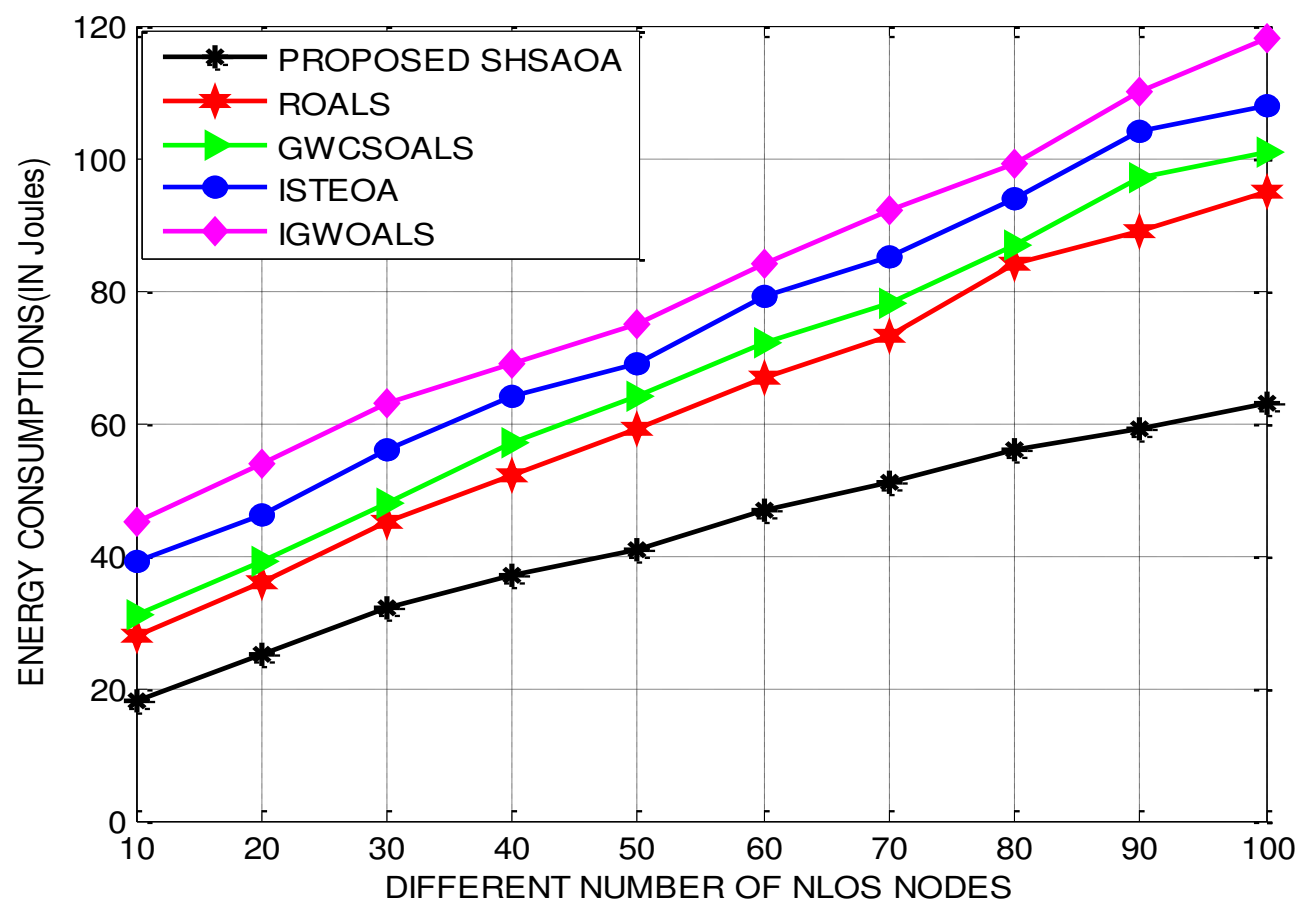

Figure 8: Proposed SHSAOA: Energy Consumptions with different NLOS vehicles 


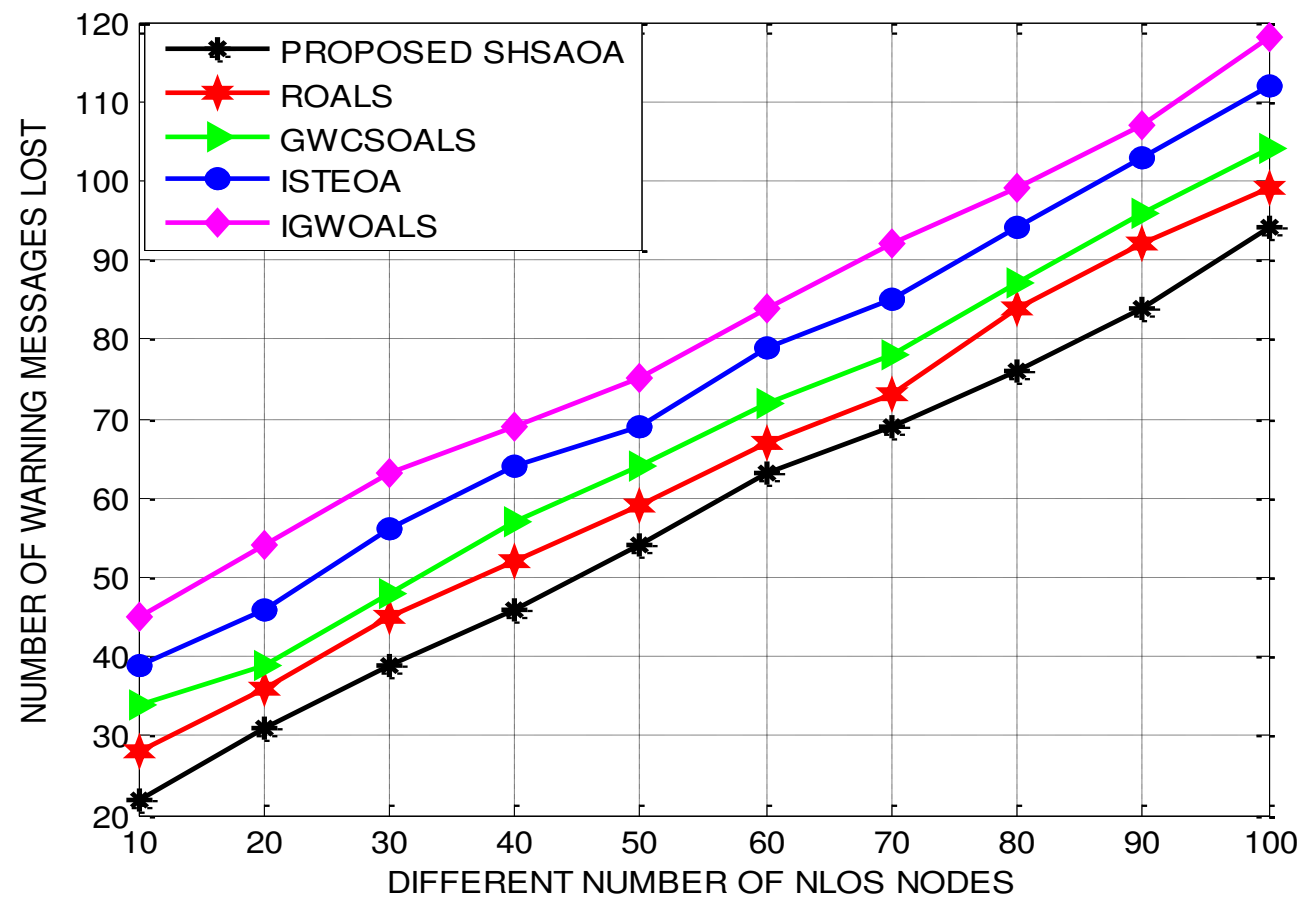

Figure 9: Proposed SHSAOA: Number of warning messages lost with different NLOS vehicles

Figure 8 and 9 depicts the energy consumptions and number of warning messages lost achieved by the proposed SHSAOA over the competitive ROALS, GWCSOALS and ISTEOA localization approaches with different NLOS nodes. The energy consumptions and the number of warning messages lost by the proposed SHSAOA mechanism is determined to be comparatively better than the competitive approaches independent to the scalable increase in the number of vehicular nodes. This is mainly due to the adaptive capability of GR-ISA incorporated into SHOA for improving its exploitation tendencies that plots the best search agents which are very near close to the unknown NLOS nodes. This potential capabilities of SHSAOA prevented unnecessary energy drain during localization and lost of warning messages are considerably minimized compared to the baseline schemes. Hence, the energy consumptions of the proposed SHSAOA scheme with different vehicular nodes is identified to be improved by $8.46 \%, 9.84 \%, 10.63 \%$ and $11.69 \%$, excellent over the competitive localization approaches. The number of warning messages lost by the proposed SHSAOA scheme with different vehicular nodes is identified to be minimized by $7.12 \%, 8.32 \%, 9.83 \%$ and $10.73 \%$, significant to the competitive ROALS, GWCSOALS, ISTEOA and IGWOALS localization approaches.

\subsection{Performance Evaluation of proposed SHSAOA with different traffic intensities}

In this investigation process, the proposed SHSAOA and the competitive ROALS, GWCSOALS and ISTEOA localization approaches are compared based on overall Root Mean 
Square Error (RMSE) and overall Mean Absolute Error (MAE) with different traffic intensities varied from 240 vehicles per hour to 3600 vehicles per hour. This investigation is conducted with overall RMSE and overall MAE, since it is the commonly utiizzed metric considered for estimating the error of the NLOS localization approaches. Figure 10 and 11 portrays the overall RMSE and MAE incurred by the proposed SHSAOA over the competitive ROALS, GWCSOALS and ISTEOA localization approaches with different traffic intensities. The results depicts that the overall RMSE and MAE of the proposed SHSAOA and the benchmarked schemes decreases with respective increase in the traffic intensities, since the possibility of nearing a unknown NLOS nodes is maximized. The proposed SHSAOA is even more potent compared to the competitive schemes, since it adopted a positioning scheme for predicting the location of unknown nodes by exploring the possible dimensions that influence the position determination of NLOS nodes. Moreover, it adopted the process of reannealing that aided in better exploitation of search space resulting in reducing the error of localization during the process of positioning. Thus, the overall RMSE attained by the proposed SHSAOA scheme with different traffic intensities is proved to be considerably reduced by $6.32 \%, 7.94 \%, 8.88 \%$ and $9.76 \%$, predominant over the competitive ROALS, GWCSOALS, ISTEOA and IGWOALS localization approaches. The overall MAE achieved by the proposed SHSAOA scheme with different traffic intensities is also determined to be significantly minimized by $7.18 \%, 8.62 \%, 9.69 \%$ and $10.64 . \%$, better than the competitive localization approaches.

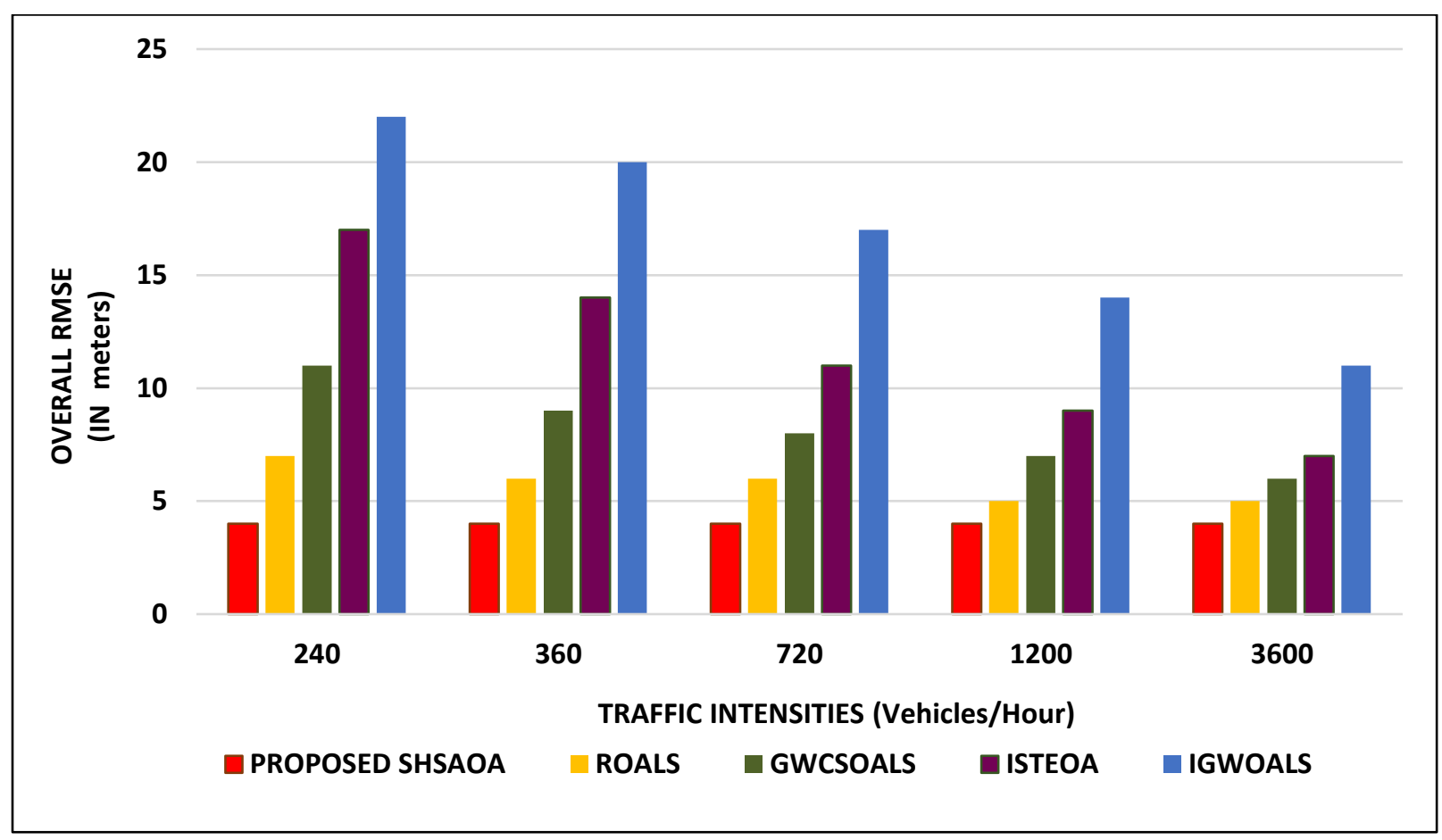

Figure 10: Proposed SHSAOA: Overall RMSE with different traffic densities 


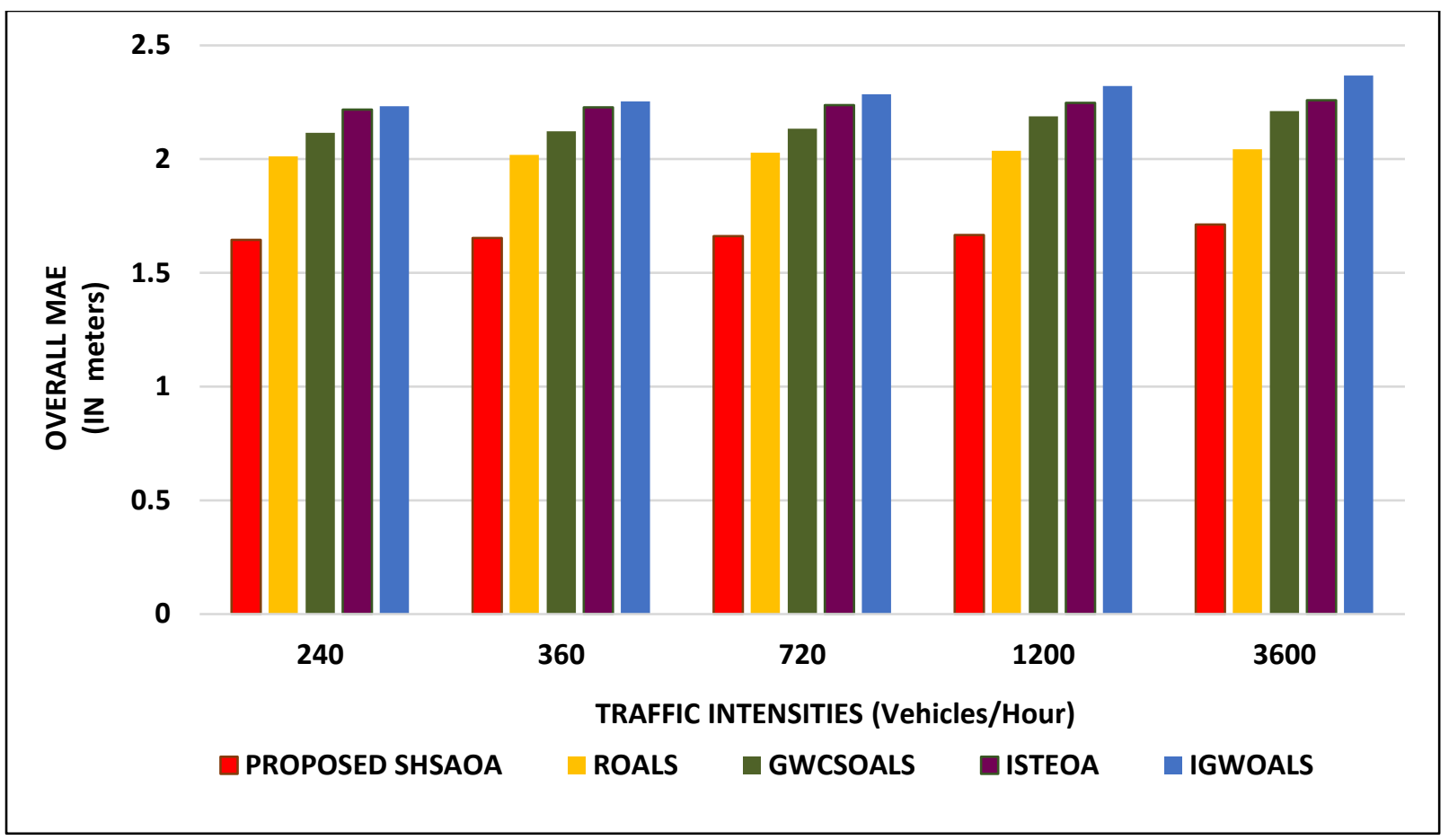

Figure 11: Proposed SHSAOA: Overall MAE with different traffic densities

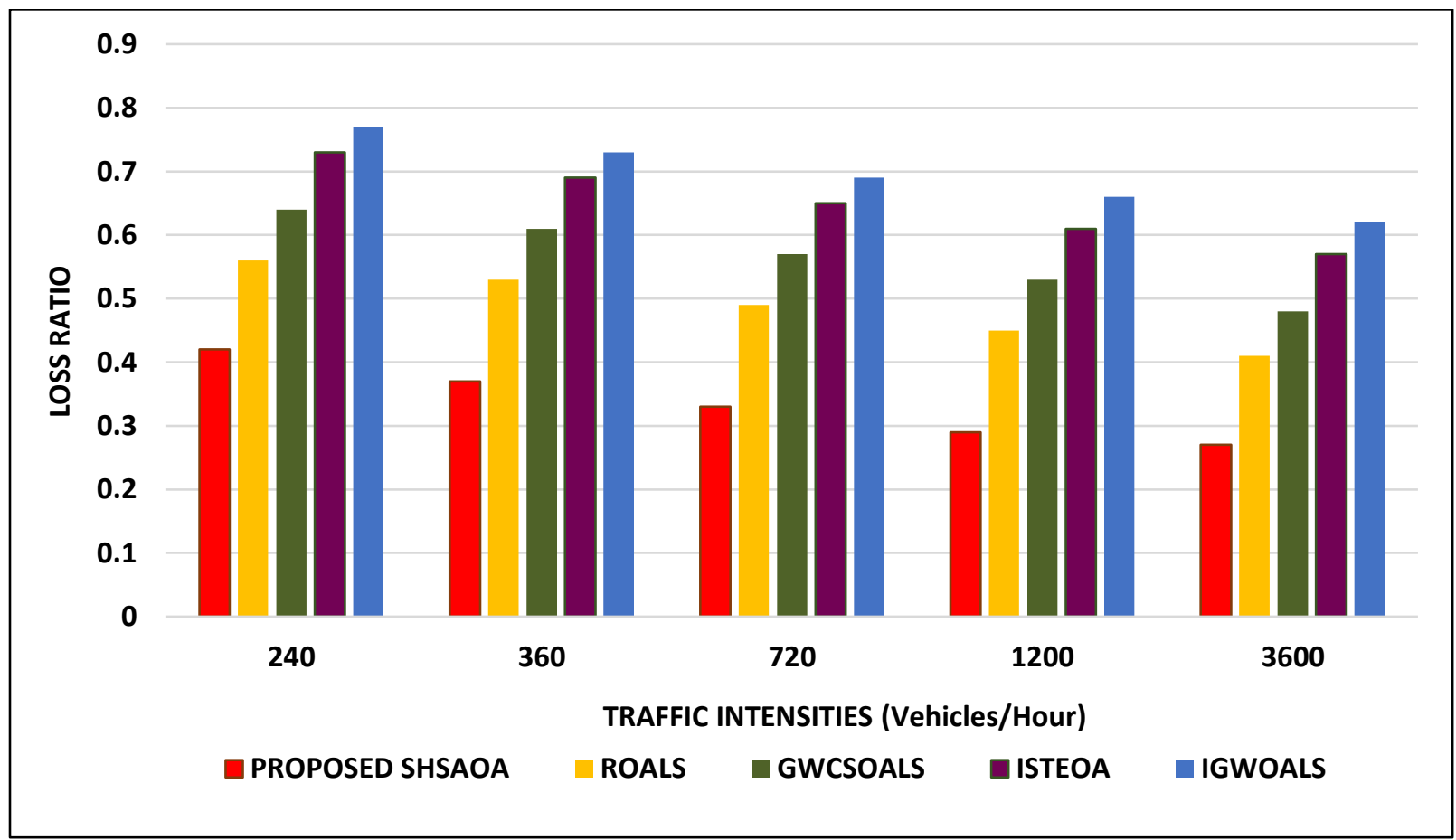

Figure 12: Proposed SHSAOA: Loss Ratio with different traffic densities 


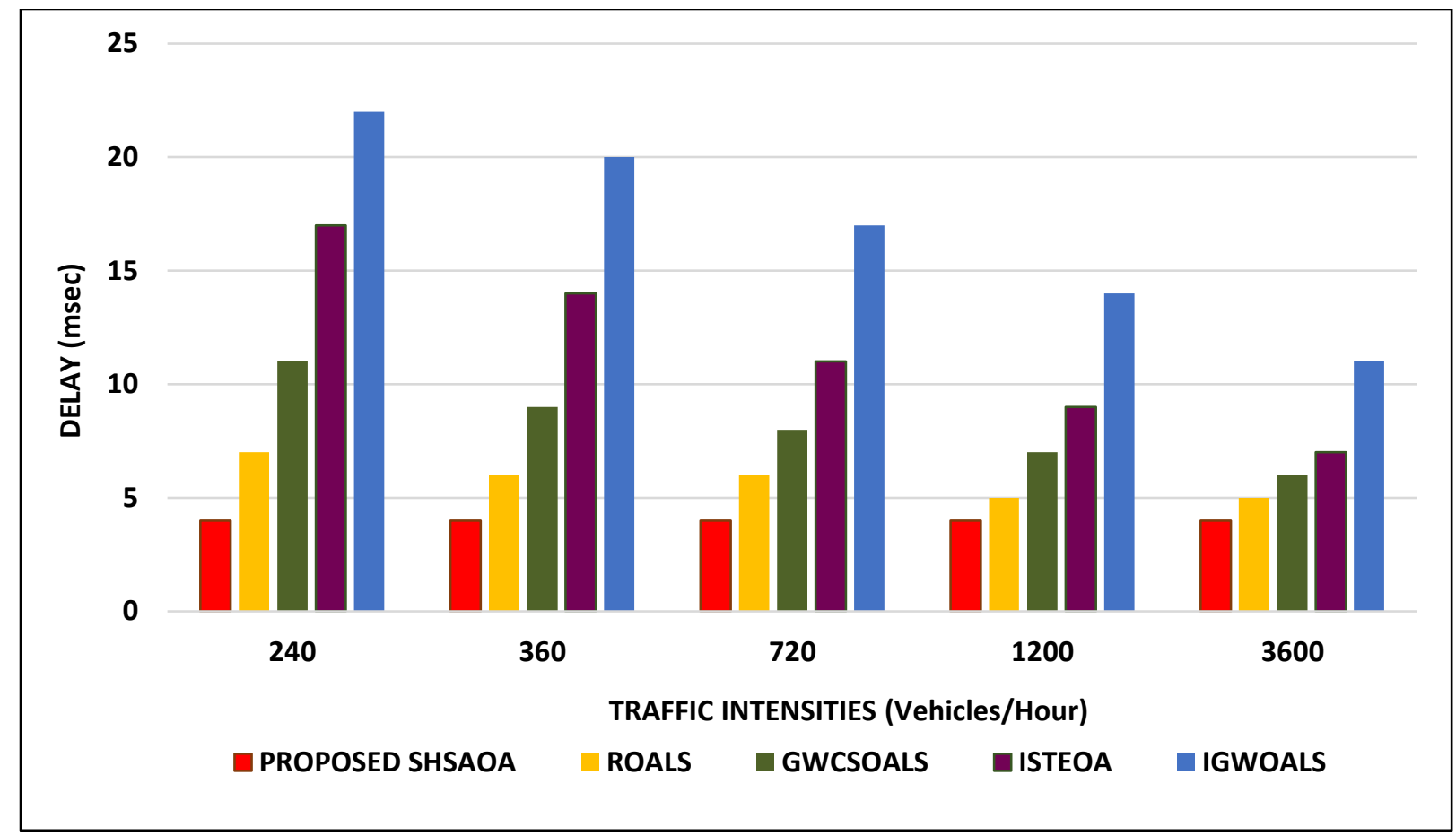

Figure 13: Proposed SHSAOA: Delay with different traffic densities

Figure 12 and 13 presents the loss ratio and delay incurred by the proposed SHSAOA over the competitive ROALS, GWCSOALS and ISTEOA localization approaches with different traffic intensities. In this investigation, the loss ratio is calculated based on the number of warning messages received by a specific destination vehicle to the number of messages actually destinated to it during emergency situations. The loss ratio of the proposed SHSAOA and the benchmarked schemes decrease systematically with corresponding increase in the traffic intensities. This is mainly due to the possibility of packet collision and interference that gets introduced with increased number of packets transmitted by the vehicles on the network. This feasibility of interference and packet collision emerging due to obstacles prevents and increases the time of packets in reaching the target vehicles during emergency. However, the proposed scheme included a dynamic updating strategy of exploration that determines the position of NLOS nodes with reduced time, thereby minimizing the loss ratio and delay. Hence, the loss ratio visualized during the implementation of the proposed SHSAOA scheme with different traffic intensities is potentially minimized by $6.81 \%, 7.58 \%, 8.62 \%$ and $9.98 \%$, predominant over the competitive ROALS, GWCSOALS, ISTEOA and IGWOALS localization approaches. The delay incurred by the proposed SHSAOA scheme with different traffic intensities is also determined to be significantly reduced by $6.42 \%, 7.34 \%, 8.86 \%$ and $9.91 \%$, better than the competitive localization approaches

\subsection{Performance Evaluation of proposed SHSAOA with different time outages}


In this performance evaluation, the proposed SHSAOA and the competitive ROALS, GWCSOALS and ISTEOA localization approaches are compared based on overall RMSE and overall MAE with increase in percentage of time outages varied from $20 \%$ to $100 \%$. Figure 14 and 15 portrays the overall RMSE and MAE incurred by the proposed SHSAOA over the competitive ROALS, GWCSOALS and ISTEOA localization approaches with different time outages. The proposed SHSAOA scheme with percentage of time outages minimized overall RMSE and MAE, since it maintained balance between the local and global search with updated search equations. Thus, the overall RMSE attained by the proposed SHSAOA scheme with different traffic intensities is proved to be considerably reduced by $7.98 \%, 8.64 \%, 9.29 \%$ and $10.42 \%$, predominant over the competitive ROALS, GWCSOALS, ISTEOA and IGWOALS localization approaches. The overall MAE achieved by the proposed SHSAOA scheme with different traffic intensities is also determined to be significantly minimized by $8.98 \%, 9.52 \%, 10.45 \%$ and 11.94.\%, better than the competitive localization approaches.

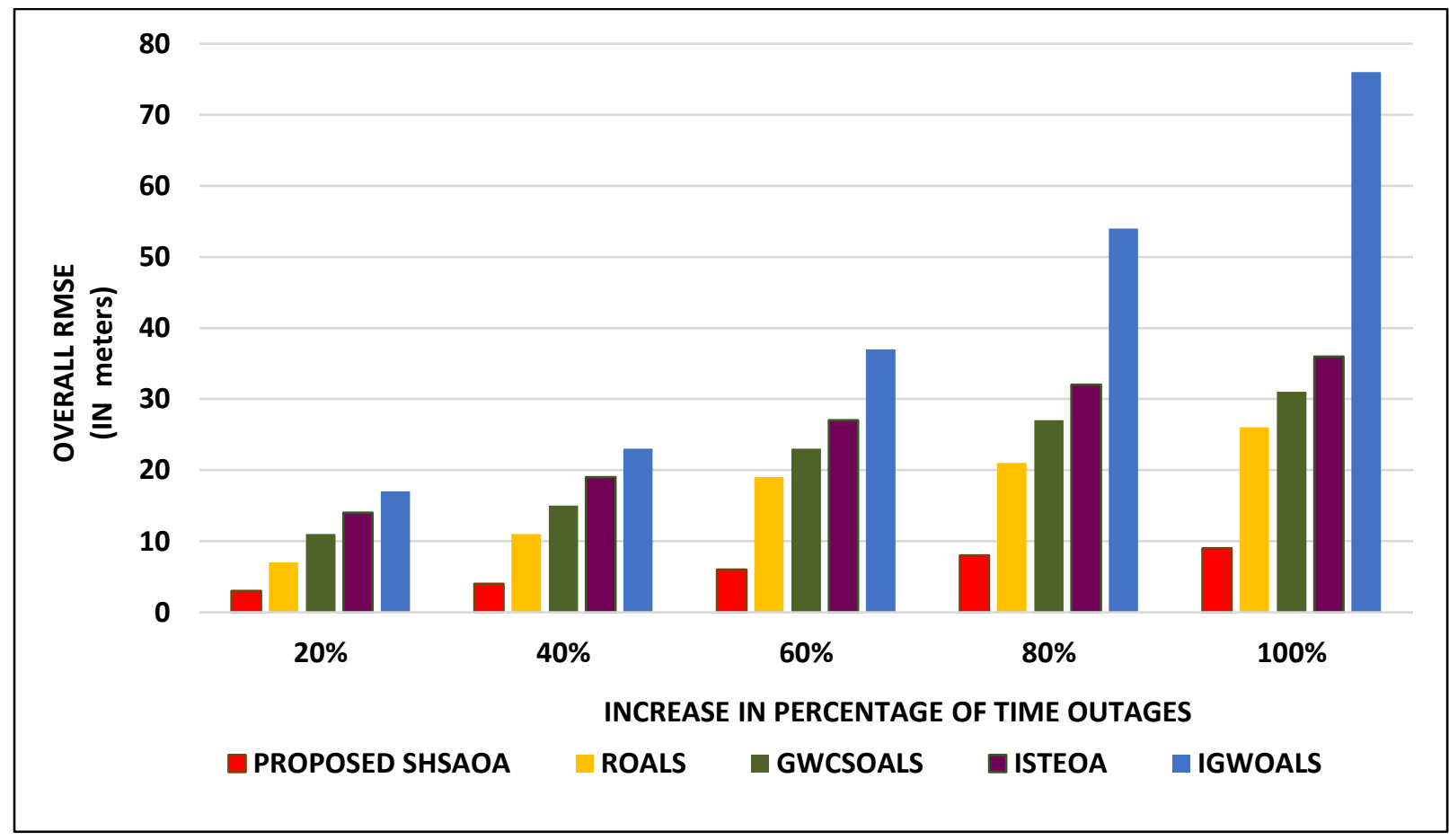

Figure 14: Proposed SHSAOA: Overall RMSE with different time outages 


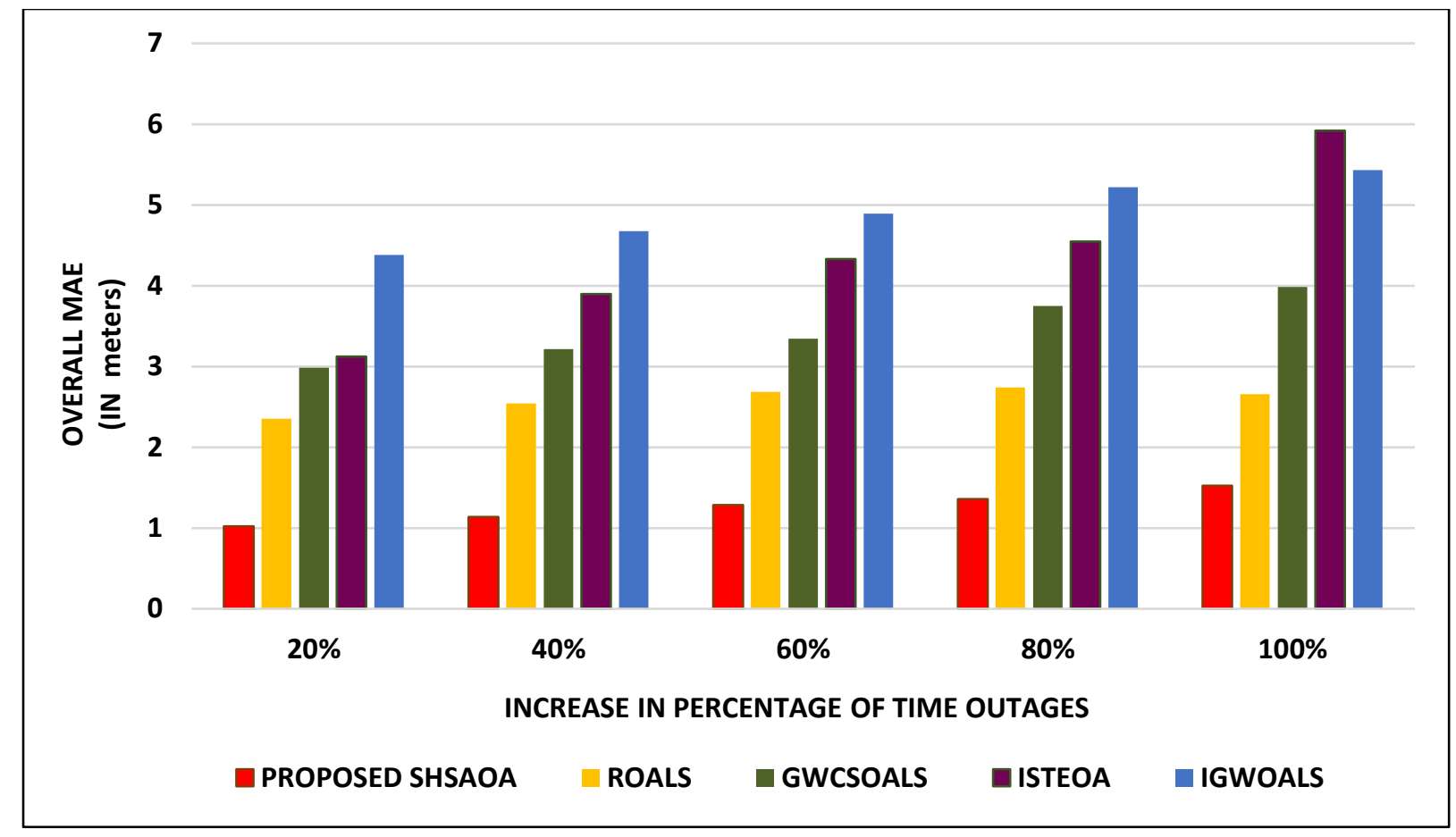

Figure 15: Proposed SHSAOA: Overall MAE with different time outages

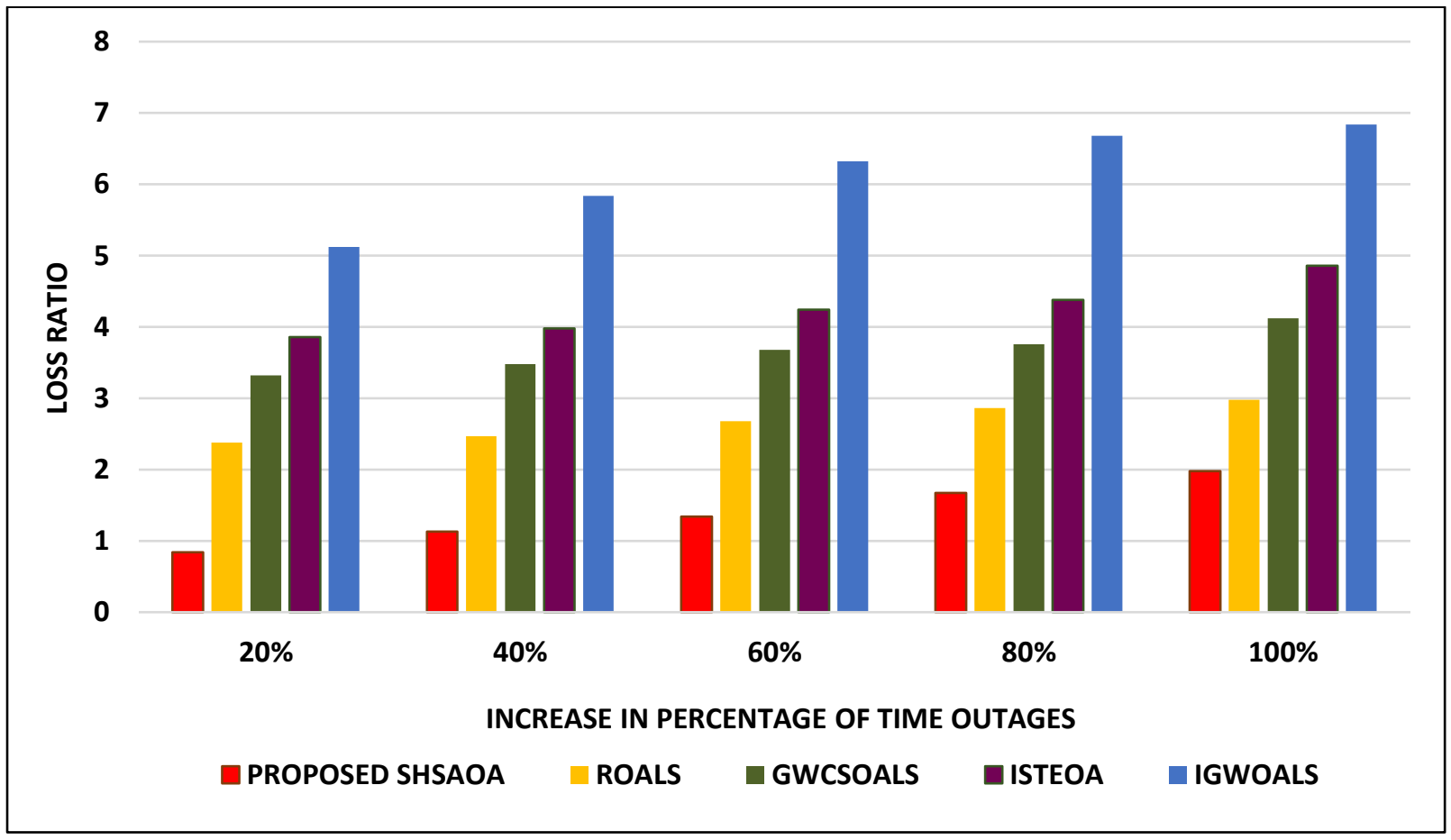

Figure 16: Proposed SHSAOA: Loss Ratio with different time outages 


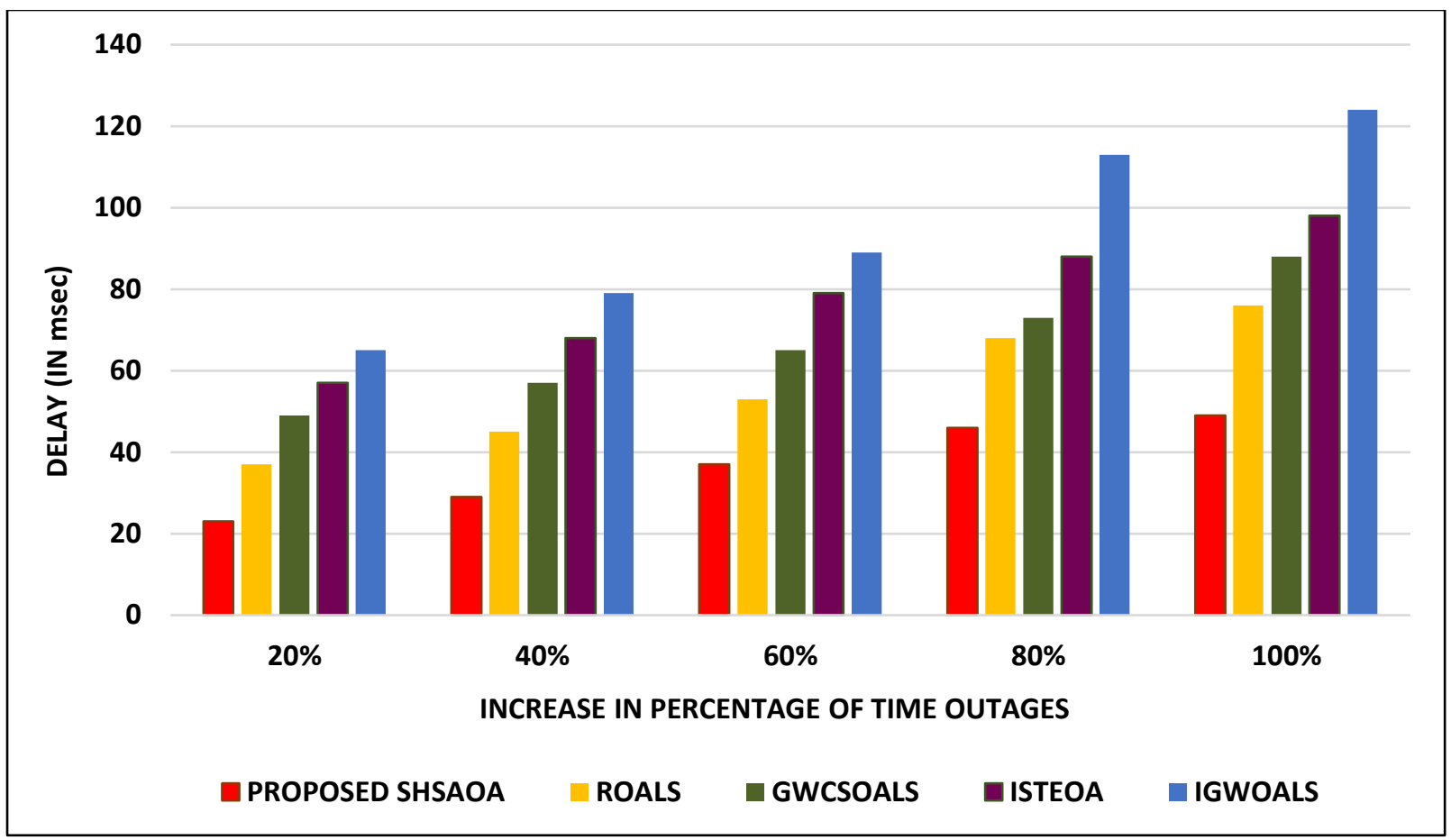

Figure 17: Proposed SHSAOA: Delay with different time outages

Figure 16 and 17 highlights and incurred by the proposed SHSAOA over the competitive ROALS, GWCSOALS and ISTEOA localization approaches with different time outages. With different increasing time outages, the loss ratio and delay of the proposed SHSAOA and baseline approaches gradually increases. But, the proposed SHSAOA is reliable enough in preventing the loss of packets during interference and aids in better routing of warning messages through the most potent link determined from the source and destination through the premier tendencies of search agents that balances exploitation and exploration to an expected level. This sustenance of exploitation and exploration degree helps in predominant identification of links that could be crumbled by obstacles during warning messages delivery in order to impose suitable reinvestigation process over them. The simulation proved that the overall RMSE and MSE attained by the proposed SHSAOA scheme with different traffic intensities is proved to be reduced, on an average by $8.27 \%$ and $9.04 \%$, predominant over the competitive ROALS, GWCSOALS, ISTEOA and IGWOALS localization approaches. The loss ratio and dealy incurred during the implementation of the proposed SHSAOA scheme with different time outages, on an average is potentially reduced $9.02 \%$ and, $10.12 \%$, better than the ompetitive ROALS, GWCSOALS, ISTEOA and IGWOALS localization approaches.

\section{Conclusions}

In this paper, the proposed SHSAOA was cooperative localization approach with the intelligent hunting and encircling characteristics of Spotted Hyena search ageny that aids in determining the difference between the previous solutions and the current solutions (location of 
nodes) for enhancing the efficiency of positioning NLOS nodes in the network. It derived the benefits of improved simulated annealing (SA) integrated into SHOA for establishing better balance between the process of exploitation and exploration in the search space. It integrated SHOA and GR-ISA algorithm for generating the candidate solutions by deriving the merits of the trajectory-based charateristics of SA throughout the algorithmic development process in order to improve the local optimization process. It also inherited reannealing for improving the nondominated solutions identified in the novel generation phase. The simulation results of the proposed SHSAOA confirmed better mean warning message rate of $17.26 \%$, mean channel utilization rate of $19.21 \%$, mean neighborhood awarness rate of $16.84 \%$ with minimized mean localization error rate of $16.54 \%$ compared to the baseline approaches under scalable increase in the number of vehicular nodes in the network. The loss ratio visualized during the implementation of the proposed SHSAOA scheme with different time outages is potentially reduced $7.82 \%, 8.56 \%$, $9.48 \%$ and $10.62 \%$, better than the ompetitive ROALS, GWCSOALS, ISTEOA and IGWOALS localization approaches. The delay incurred by the proposed SHSAOA scheme with different time outages are also confirmed to be remarkably minimized by $8.12 \%, 9.61 \%, 10.98 \%$ and $11.54 \%$, better than the competitive localization approaches. As a part of future plan, it is planned to devise a Hybrid Artificial Bee Colony and Differential Evolution Optimization Algorithm-based NLOS localization scheme and compare it with the proposed SHSAOA positioning algorithm.

\section{Data Availability Statement}

Data sharing not applicable - no new data generated, Data sharing is not applicable to this article as no new data were created or analyzed in this study.

\section{Funding Information}

There is no funding received for this research work

\section{Conflict of Interest Statement}

The authors declare that there is no competing interest

\section{References}

1) Cruz, S. B., Abrudan, T. E., Xiao, Z., Trigoni, N., \& Barros, J. (2017). Neighbor-aided localization in vehicular networks. IEEE Transactions on Intelligent Transportation Systems, 18(10), 2693-2702.

2) Tripathi, R., Prakash, A., \& Gupta, N. (2017). Clustering-based enhanced safety message dissemination medium access control protocol for vehicular ad hoc network. International Journal of Ad Hoc and Ubiquitous Computing, 24(1/2), 76.

3) Singh, B., Kavitha, P., Regin, R., Praghash, D., Sujatha, S., \& Rajest, D. S. (2020). Optimized node clustering based on received signal strength with particle ordered-filter routing used in VANET. Webology, 17(2), 262-277. 
4) Soleymani, S. A., Goudarzi, S., Anisi, M. H., Kama, N., Adli Ismail, S., Azmi, A., Zareei, M., \& Hanan Abdullah, A. (2020). A trust model using edge nodes and a cuckoo filter for securing VANET under the NLoS condition. Symmetry, 12(4), 609.

5) Zhao, J., Zhang, Y., Ni, S., \& Li, Q. (2020). Bayesian cooperative localization with NLOS and malicious vehicle detection in GNSS-challenged environments. IEEE Access, 8(3), 85686-85697.

6) Ansari, A. R., Saeed, N., Ul Haq, M. I., \& Cho, S. (2018). Accurate 3D localization method for public safety applications in vehicular ad-hoc networks. IEEE Access, 6(2), 20756-20763.

7) Hu, N., Wu, C., Liu, P., Wu, H., Wu, B., \& Cheng, L. (2015). Vote selection mechanisms and probabilistic data association-based mobile node localization algorithm in mixed LOS/NLOS environments. Telecommunication Systems, 62(4), 641-655.

8) Amuthan, A., \& Kaviarasan, R. (2018). Weighted distance hyperbolic prediction-based detection scheme for non line of sight nodes in VANETs. Journal of King Saud University - Computer and Information Sciences, 2(1), 45-56.

9) Nascimento, P., Kimura, B., Guidoni, D., \& Villas, L. (2018). An integrated dead reckoning with cooperative positioning solution to assist GPS NLOS using vehicular communications. Sensors, 18(9), 2895.

10) Janakiraman, S. (2020). An improved rank criterion-based NLOS node detection mechanism in VANETs. International Journal of Intelligent Unmanned Systems, 9(1), 115.

11) Min, H., Wu, X., Cheng, C., \& Zhao, X. (2019). Kinematic and dynamic vehicle modelassisted global positioning method for autonomous vehicles with low-cost GPS/Camera/invehicle sensors. Sensors, 19(24), 5430.

12) Zubairu, B. (2017). Novel approach of spoofing attack in VANET location verification for non-line-of-Sight (NLOS). Innovations in Computational Intelligence, 2(1), 45-59.

13) Dhiman, G., \& Kumar, V. (2017). Spotted hyena optimizer: A novel bio-inspired based metaheuristic technique for engineering applications. Advances in Engineering Software, 114(2), 48-70.

14) Huang, Z., Lin, Z., Zhu, Z., \& Chen, J. (2020). An improved simulated annealing algorithm with excessive length penalty for fixed-outline Floorplanning. IEEE Access, 8(1), 50911-50920.

15) Jia, H., Li, J., Song, W., Peng, X., Lang, C., \& Li, Y. (2019). Spotted hyena optimization algorithm with simulated annealing for feature selection. IEEE Access, 7(2), 71943-71962.

16) Soleymani, S. A., Abdullah, A. H., Zareei, M., Anisi, M. H., Vargas-Rosales, C., Khurram Khan, M., \& Goudarzi, S. (2017). A secure trust model based on fuzzy logic in vehicular ad hoc networks with fog computing. IEEE Access, 5(3), 15619-15629.

17) Lobo, F., Grael, D., Oliveira, H., Villas, L., Almehmadi, A., \& El-Khatib, K. (2019). Cooperative localization improvement using distance information in vehicular ad hoc networks. Sensors, 19(23), 5231.

18) Alodadi, K., Al-Bayatti, A. H., \& Alalwan, N. (2017). Cooperative volunteer protocol to detect non-line of sight nodes in vehicular ad hoc networks. Vehicular Communications, 9(2), 72-82.

19) Amuthan, A., \& Kaviarasan, R. (2018). Weighted inertia-based dynamic virtual bat algorithm to detect NLOS nodes for reliable data dissemination in VANETs. Journal of Ambient Intelligence and Humanized Computing, 10(11), 4603-4613. 
20) Soleymani, S. A., $\quad$ Anisi, M. H., $\quad$ Abdullah, A. H., $\quad$ Ngadi, M. A., $\quad$ Goudarzi, S., Khan, M. K., \& Kama, M. N. (2020). An authentication and plausibility model for big data analytic under LOS and NLOS conditions in 5G-VANET. Science China Information Sciences, 63(12), 45-58.

21) A., Christy Jebamalar, Priya, M. D, \& Janakiraman, S. (2020). Harris Hawk Optimization Algorithm-based Effective Localization of Non-Line-of-Sight Nodes for Reliable Data Dissemination in Vehicular Ad hoc Networks, International Journal of Communication Systems, 32(2), 65-78. .

22) Balamurugan, A., Priya, M. D., Malar, A. C., \& Janakiraman, S. (2021). Raccoon optimization algorithm-based accurate positioning scheme for reliable emergency data dissemination under NLOS situations in VANETs. Journal of Ambient Intelligence and Humanized Computing, 2(1), 56-72.

23) Kaviarasan, R, Harikrishna, P. (2020). Localizing non-line-of-sight nodes in Vehicluar Adhoc Networks using gray wolf methodology. International Journal of Communication Systems, 34(3), 88-99. .

24) A., Christy Jebamalar, Priya, M. D, \& Janakiraman, S. (2020). A hybrid crow search and gray wolf optimization algorithm-based reliable non-line-of-Sight node positioning scheme for vehicular ad hoc networks. International Journal of Communication Systems, 34(3), 23-35.

25) Mani, R., Jayaraman, S., \& Ellappan, M. (2020). Hybrid seagull and thermal exchange optimization algorithm-based NLOS nodes detection technique for enhancing reliability under data dissemination in VANETs. International Journal of Communication Systems, 33(14), e4519. 


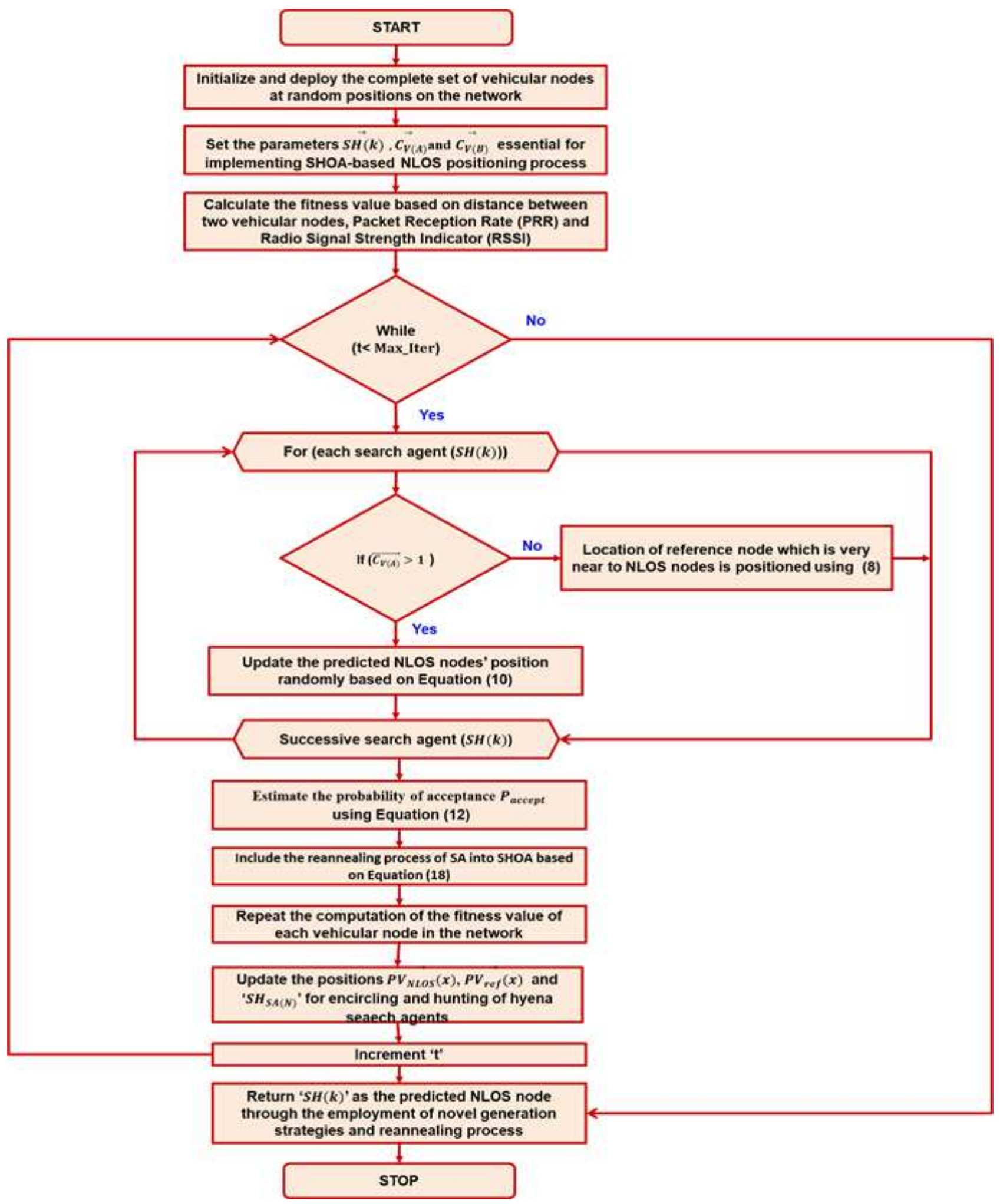

\section{Figure 1}




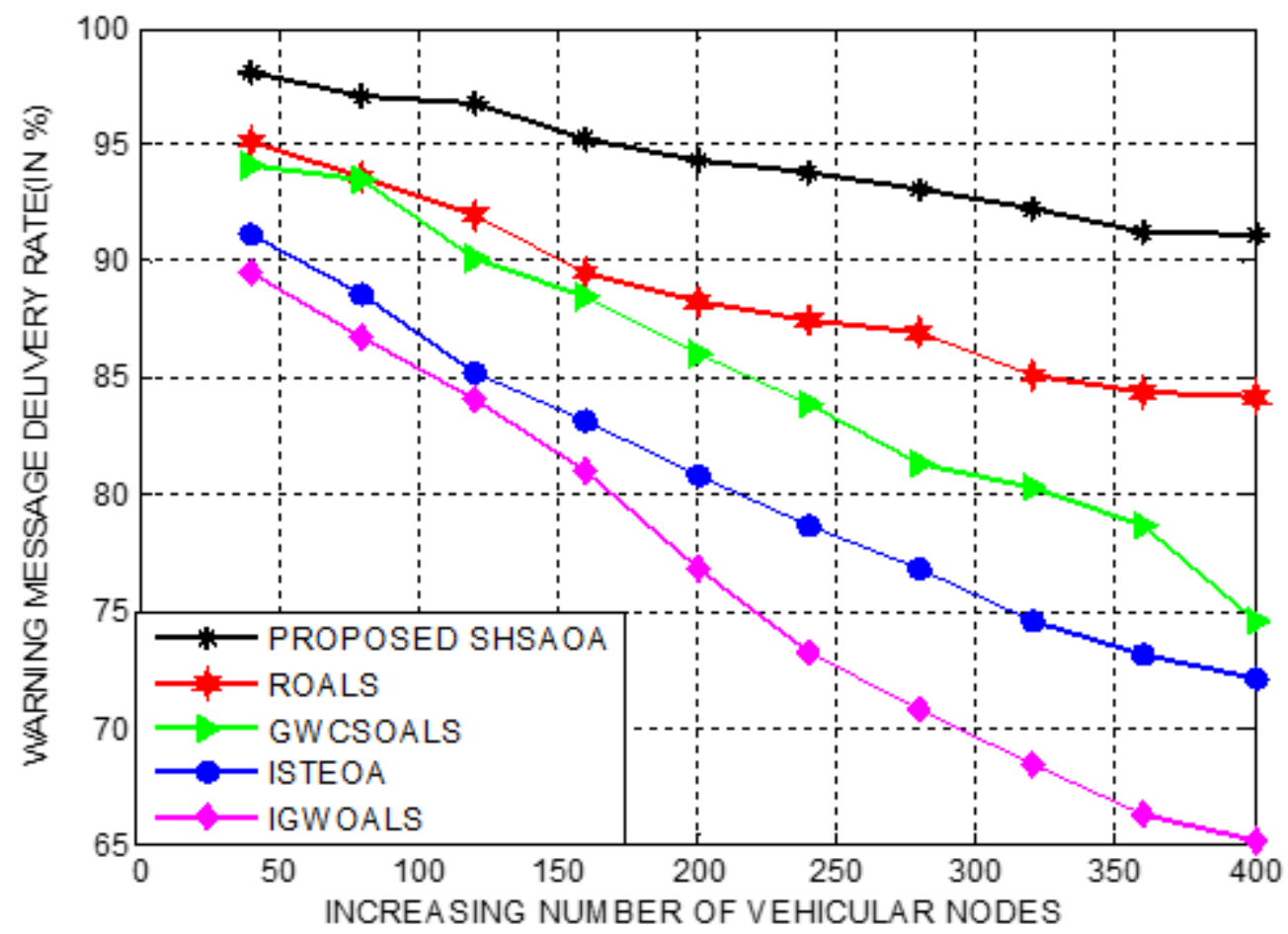

Figure 2

Proposed SHSAOA: Warning message Delivery Rate with different vehicles

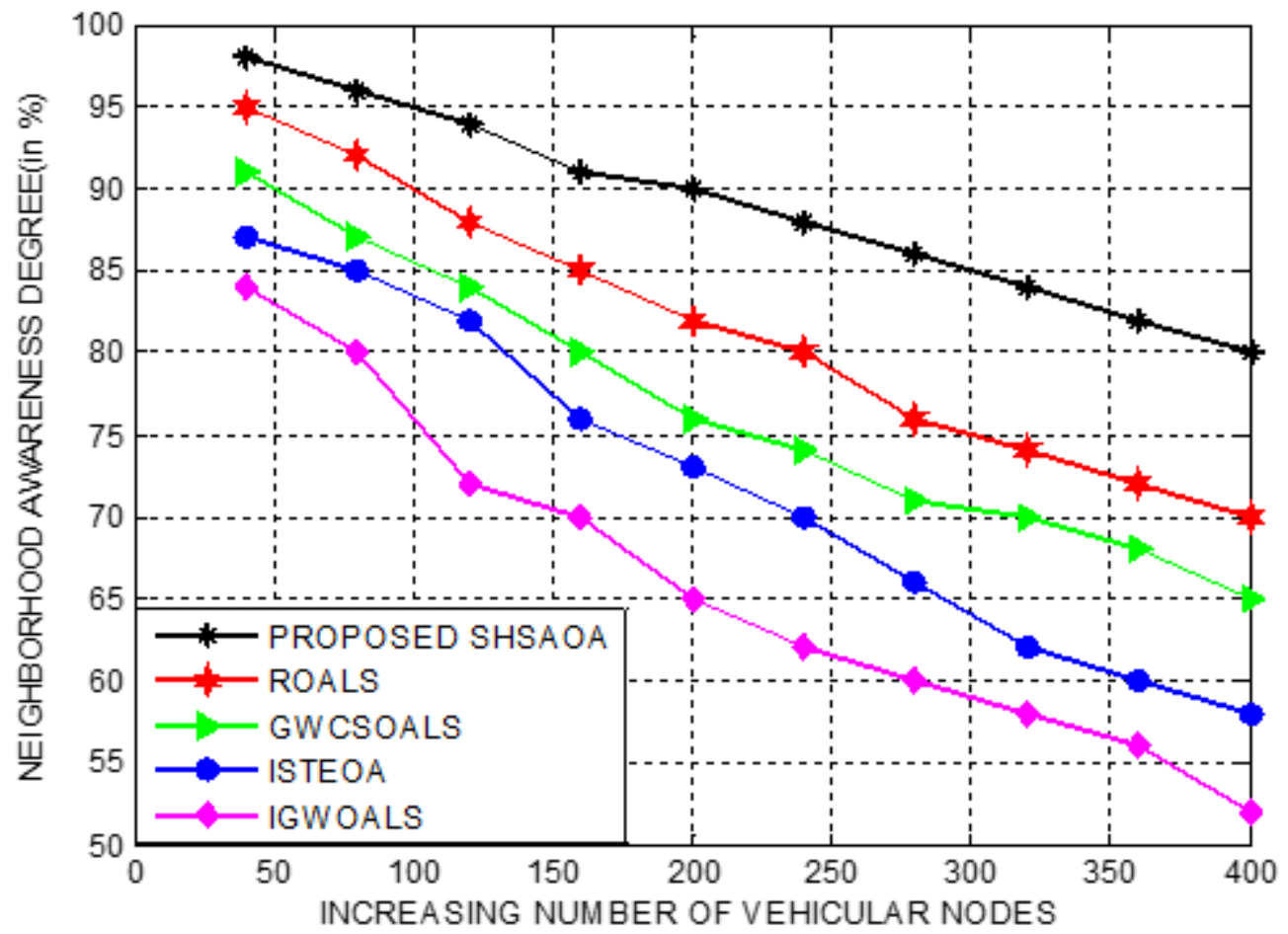

Figure 3

Proposed SHSAOA: Neighborhood Awareness Rate with different vehicles 


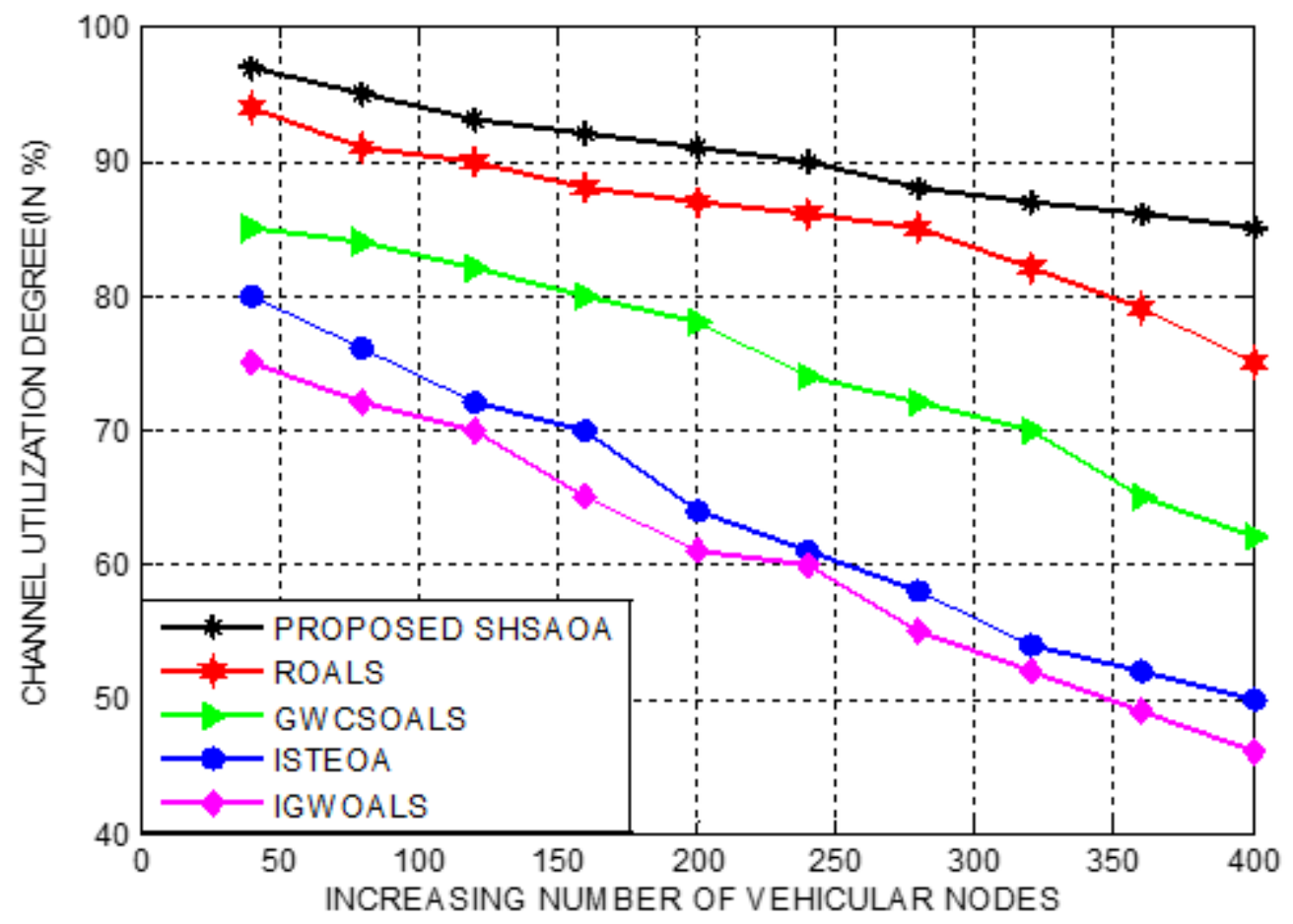

Figure 4

Proposed SHSAOA: Chanel Utilization Degree with different vehicles

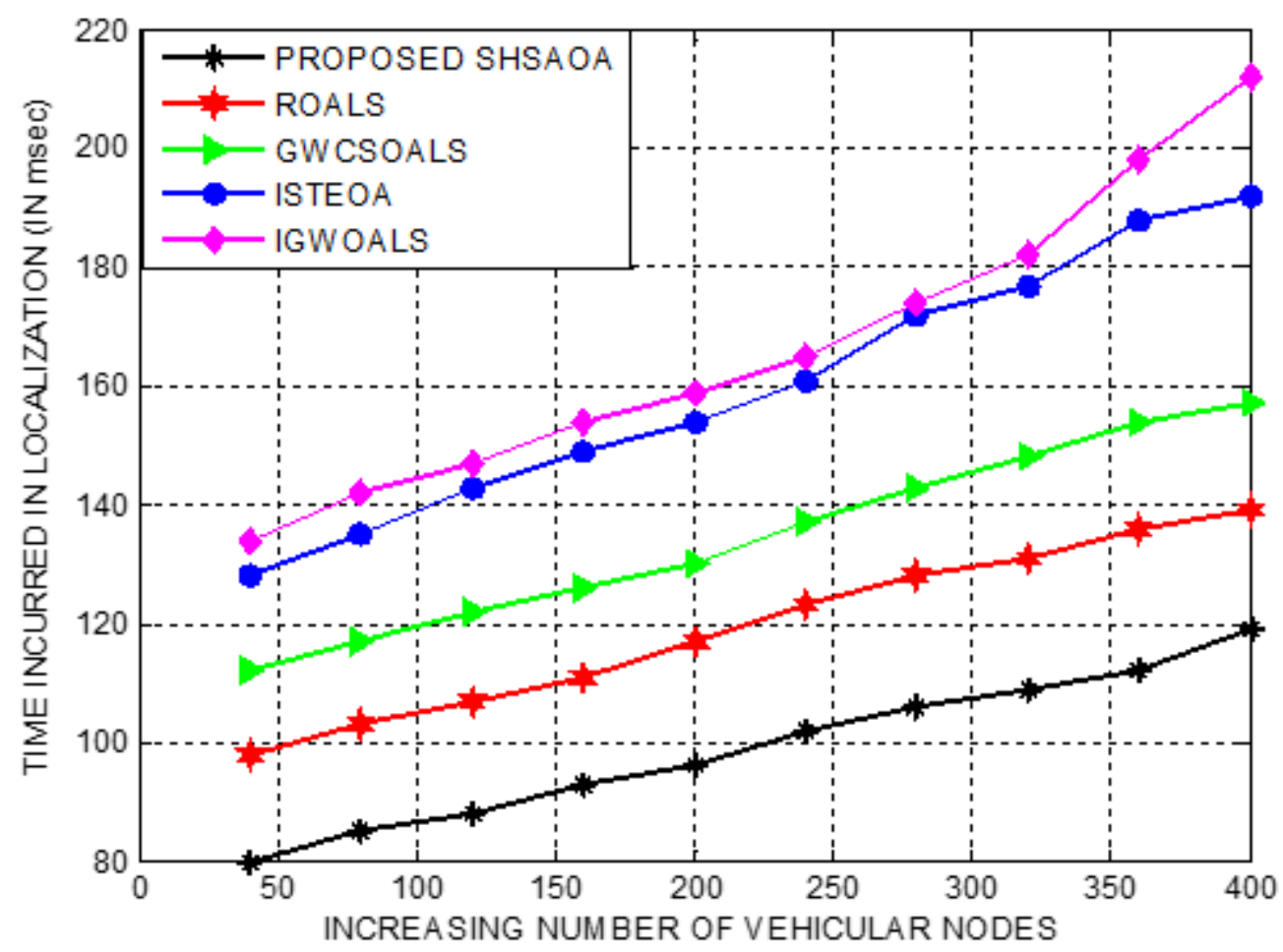

Figure 5

Proposed SHSAOA: Time incurred in localization with different vehicles 


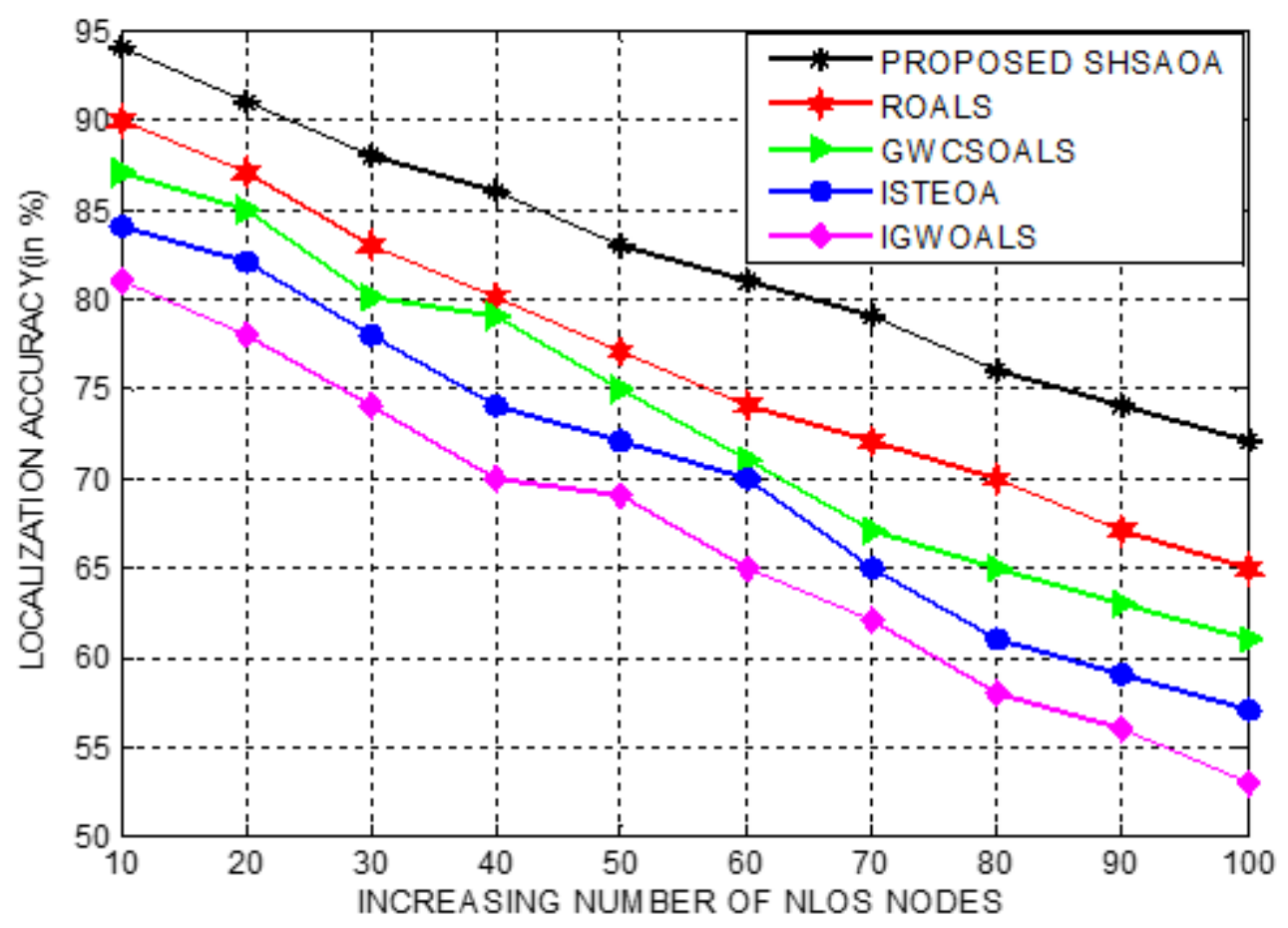

Figure 6

Proposed SHSAOA: Localization Accuracy with different NLOS vehicles

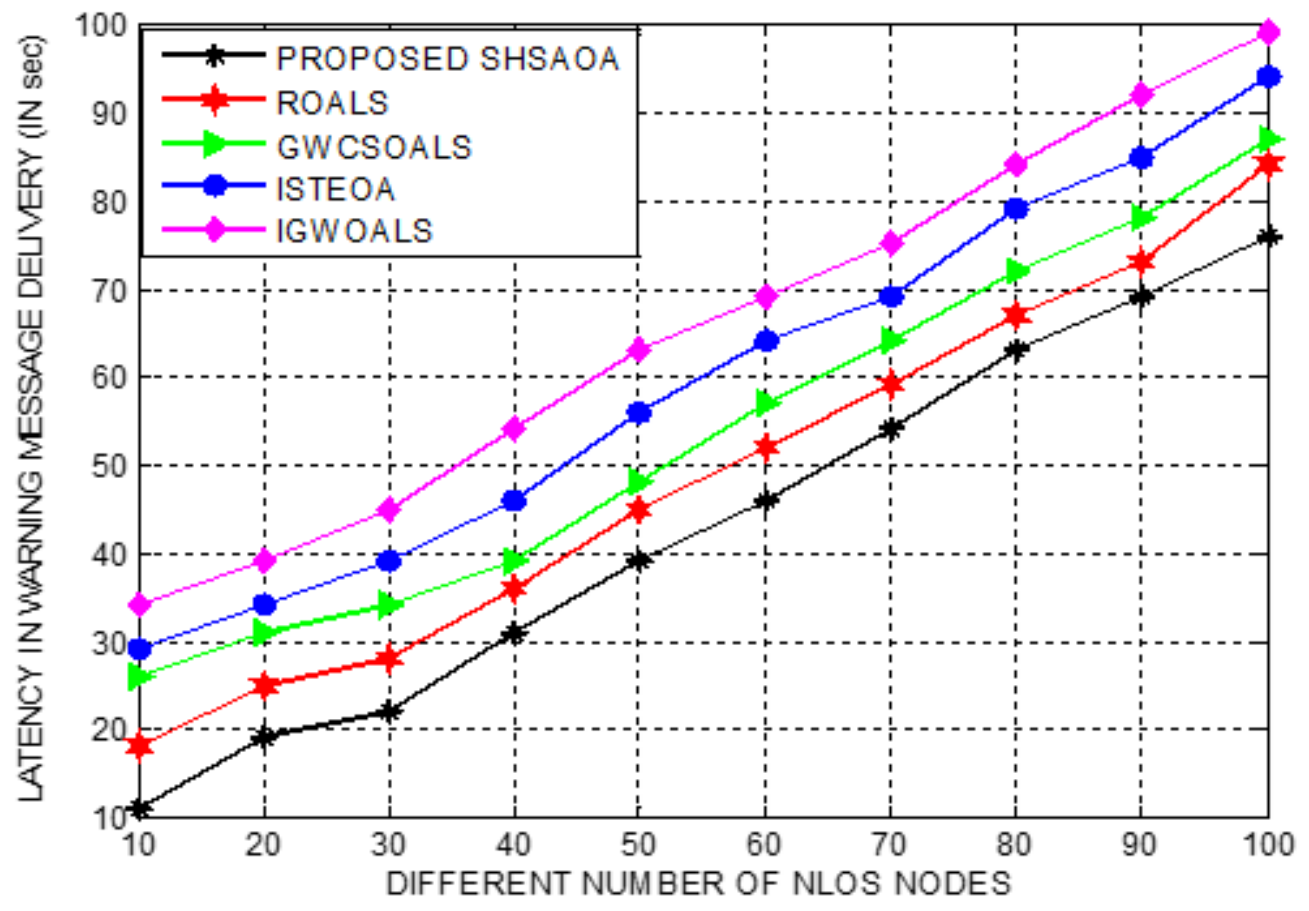

Figure 7

Proposed SHSAOA: Latency incurred during warning meassge delivery with different NLOS vehicles 


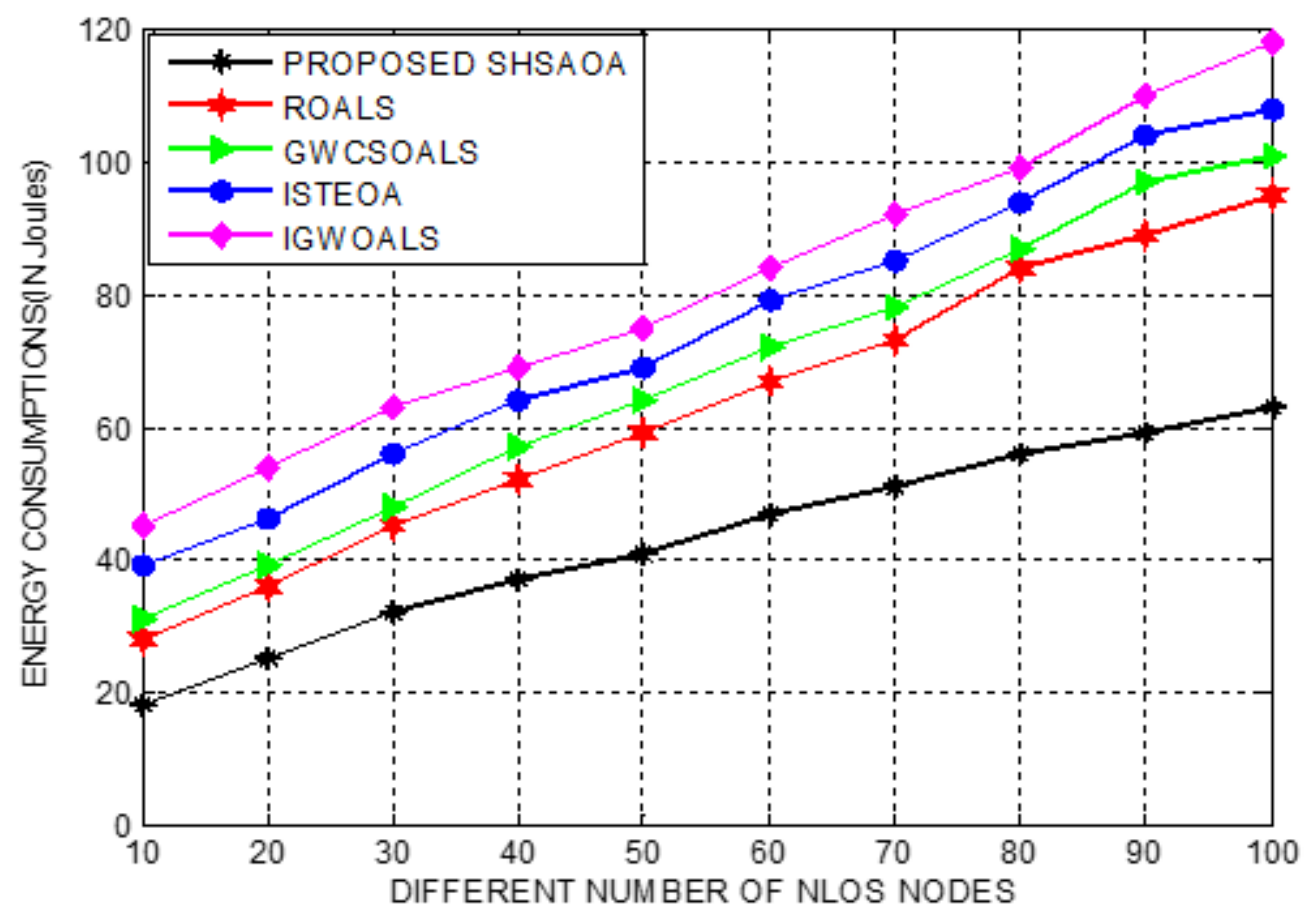

Figure 8

Proposed SHSAOA: Energy Consumptions with different NLOS vehicles

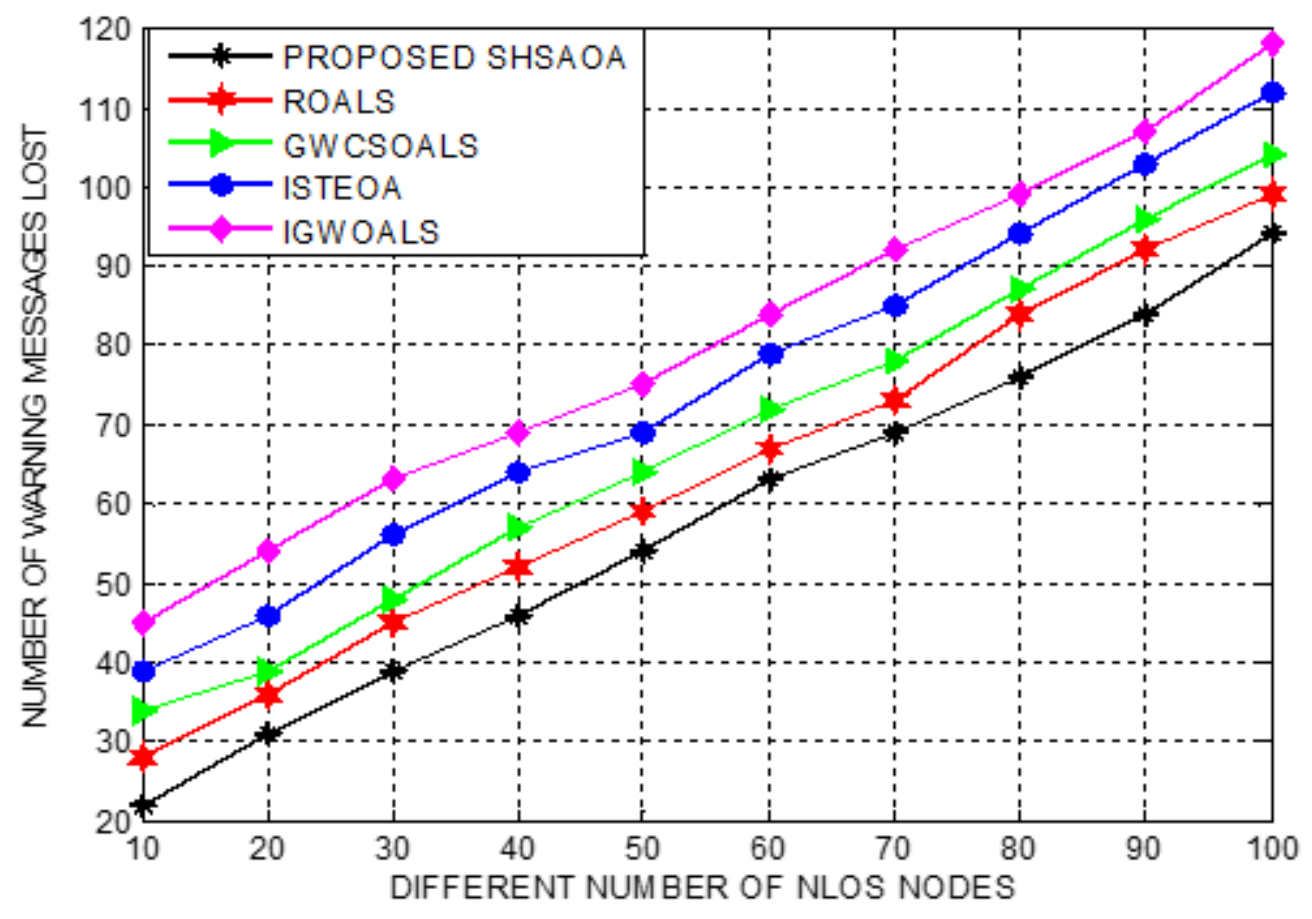

Figure 9

Proposed SHSAOA: Number of warning messages lost with different NLOS vehicles 


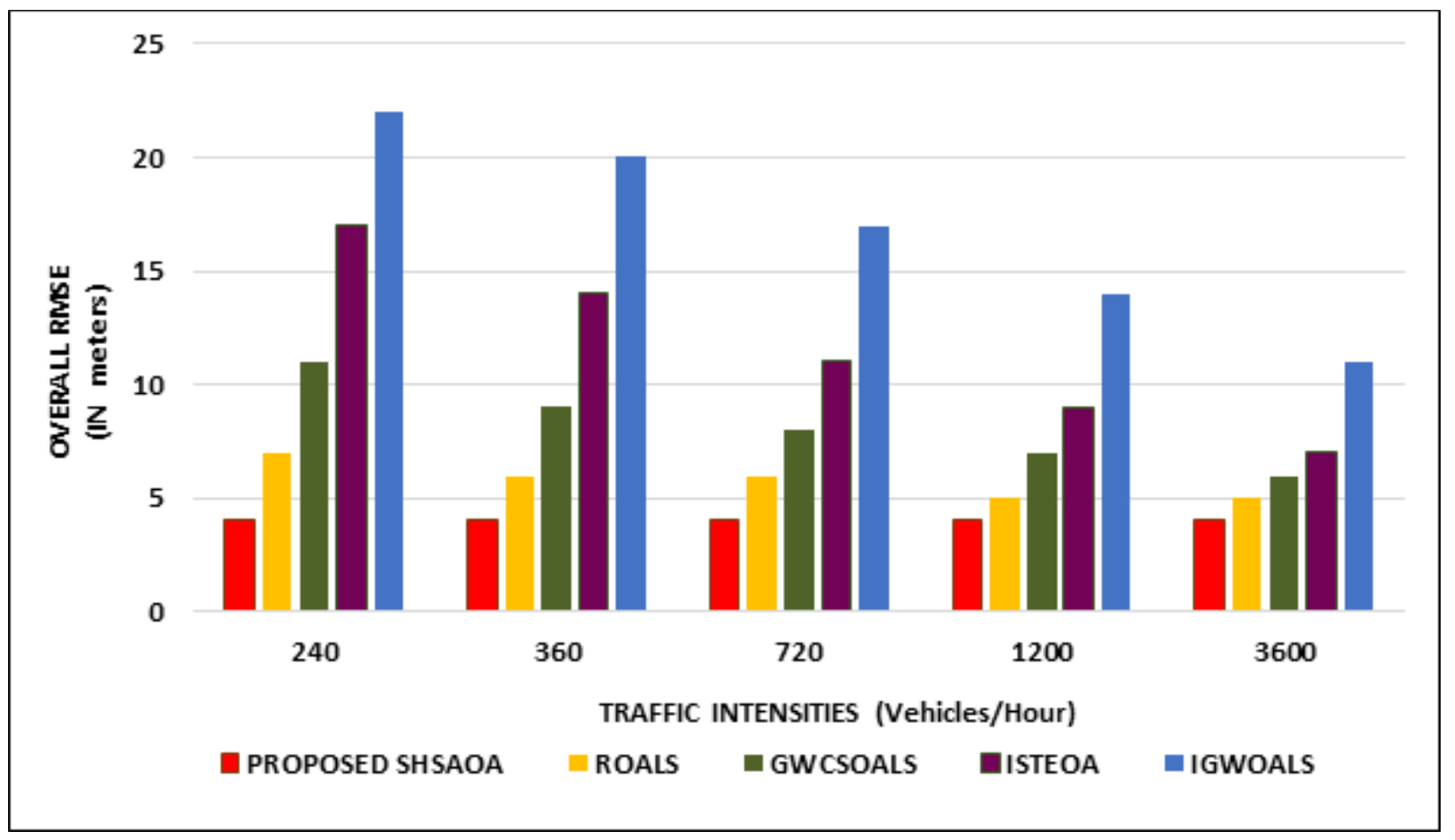

Figure 10

Proposed SHSAOA: Overall RMSE with different traffic densities

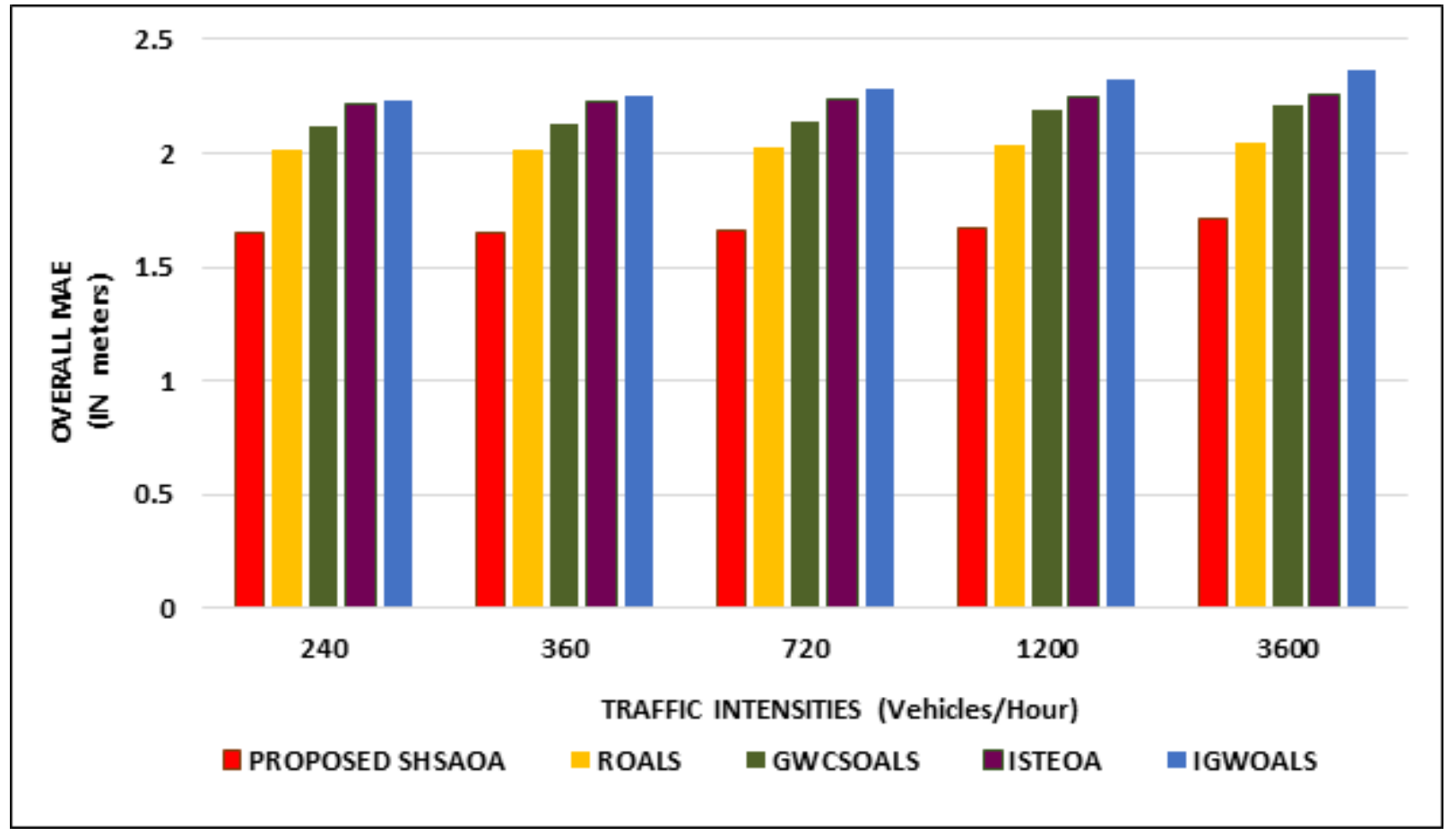

Figure 11

Proposed SHSAOA: Overall MAE with different traffic densities 


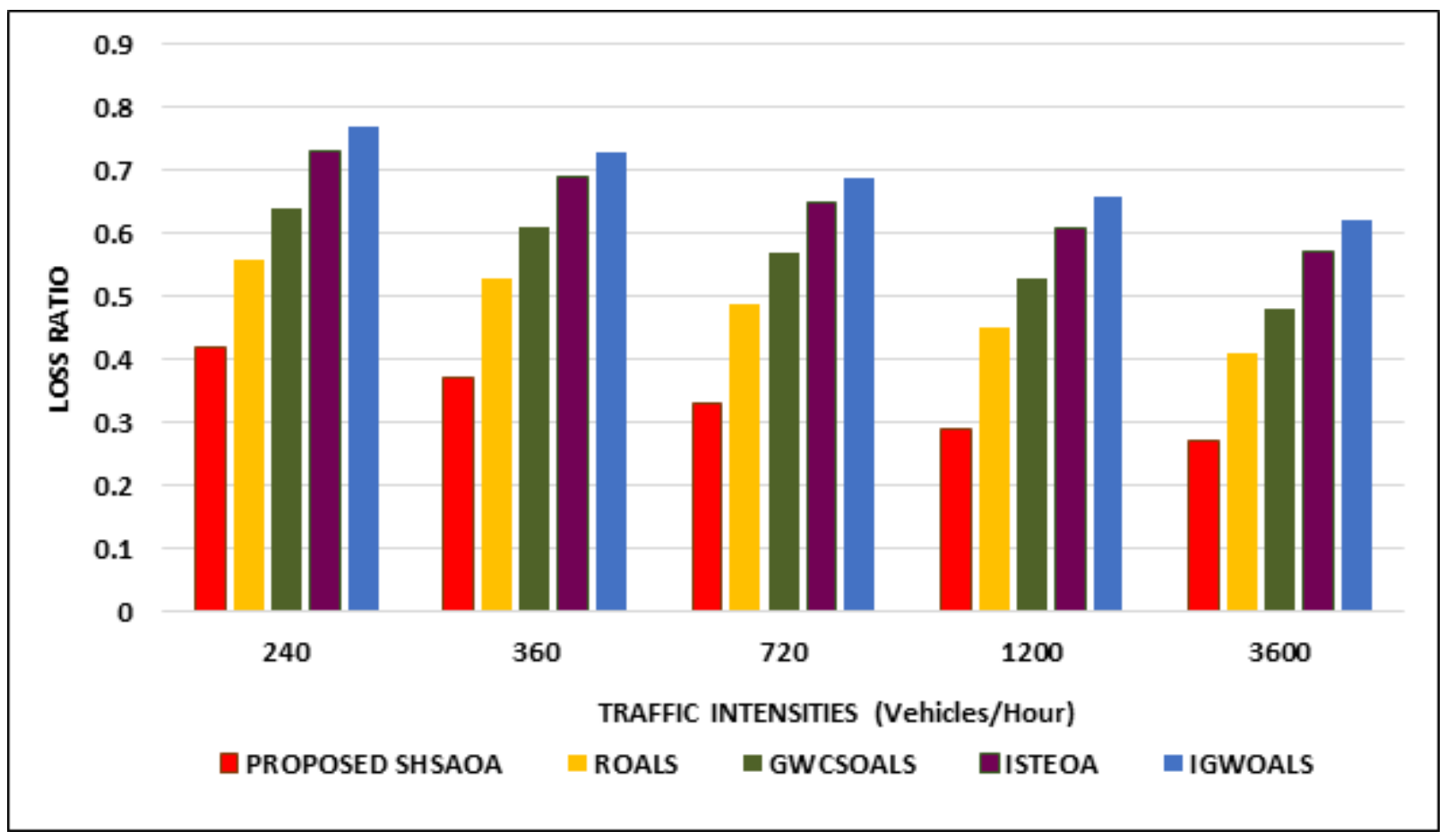

Figure 12

Proposed SHSAOA: Loss Ratio with different traffic densities

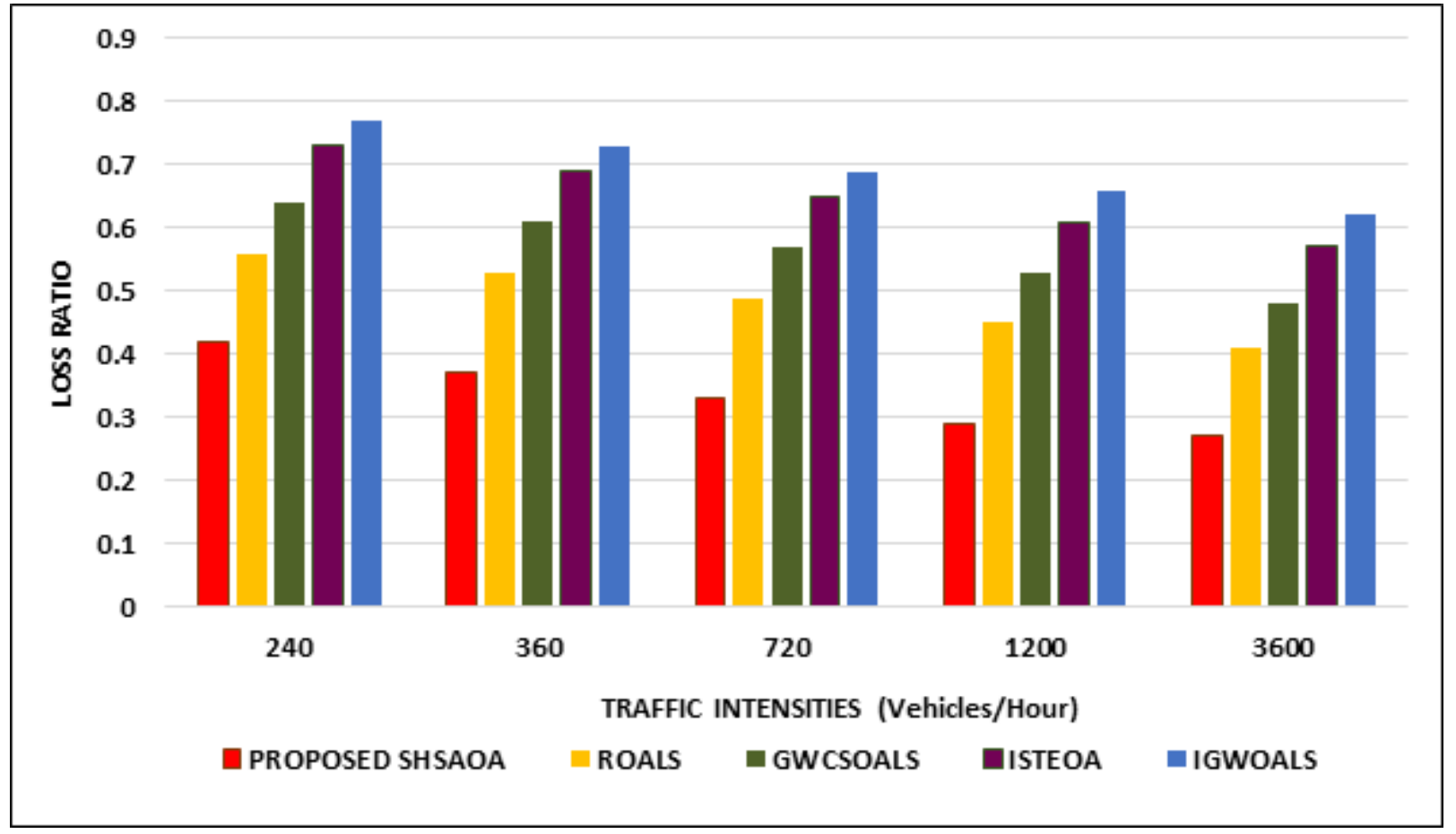

Figure 13

Proposed SHSAOA: Delay with different traffic densities 


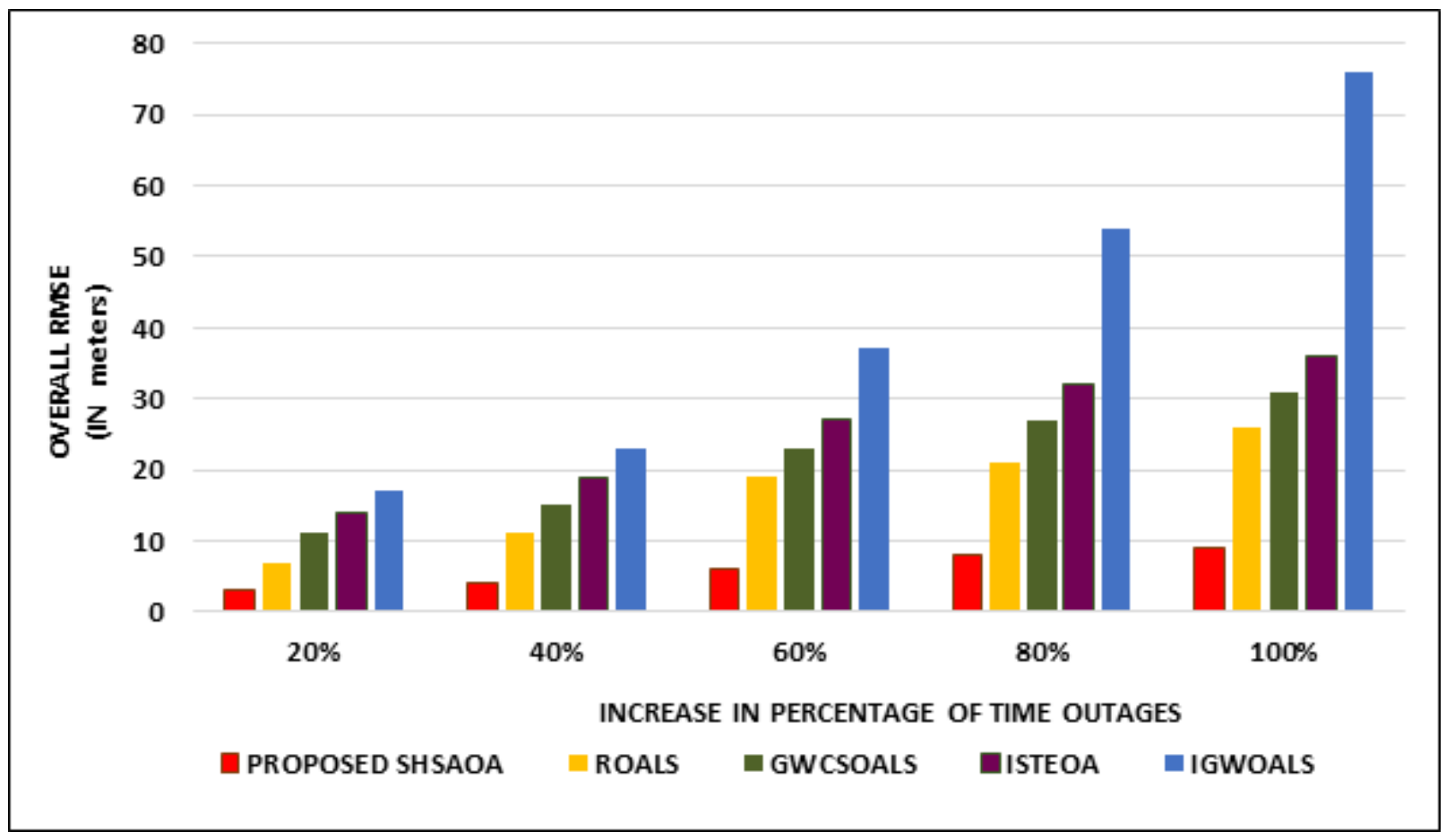

Figure 14

Proposed SHSAOA: Overall RMSE with different time outages

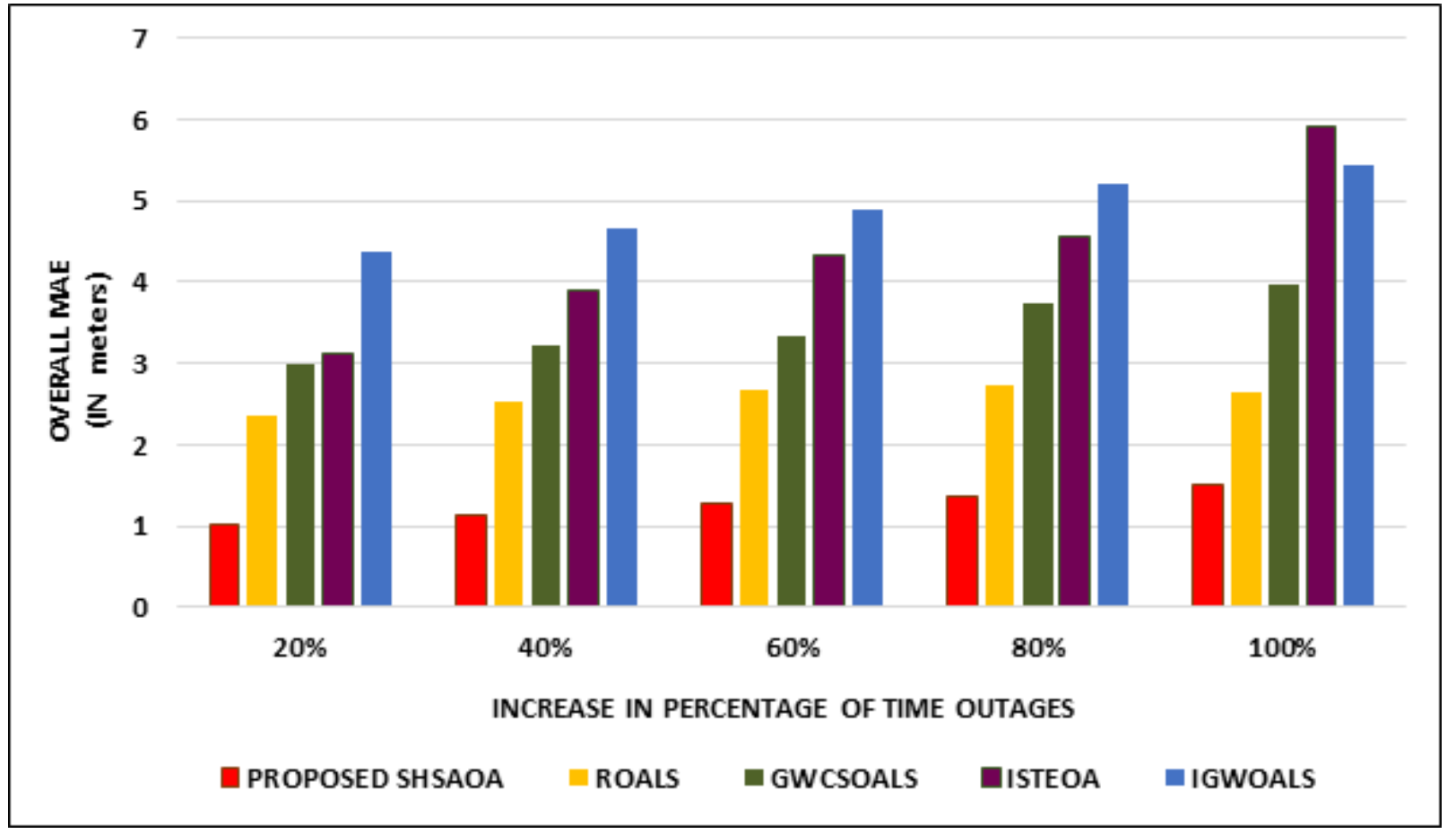

Figure 15

Proposed SHSAOA: Overall MAE with different time outages 


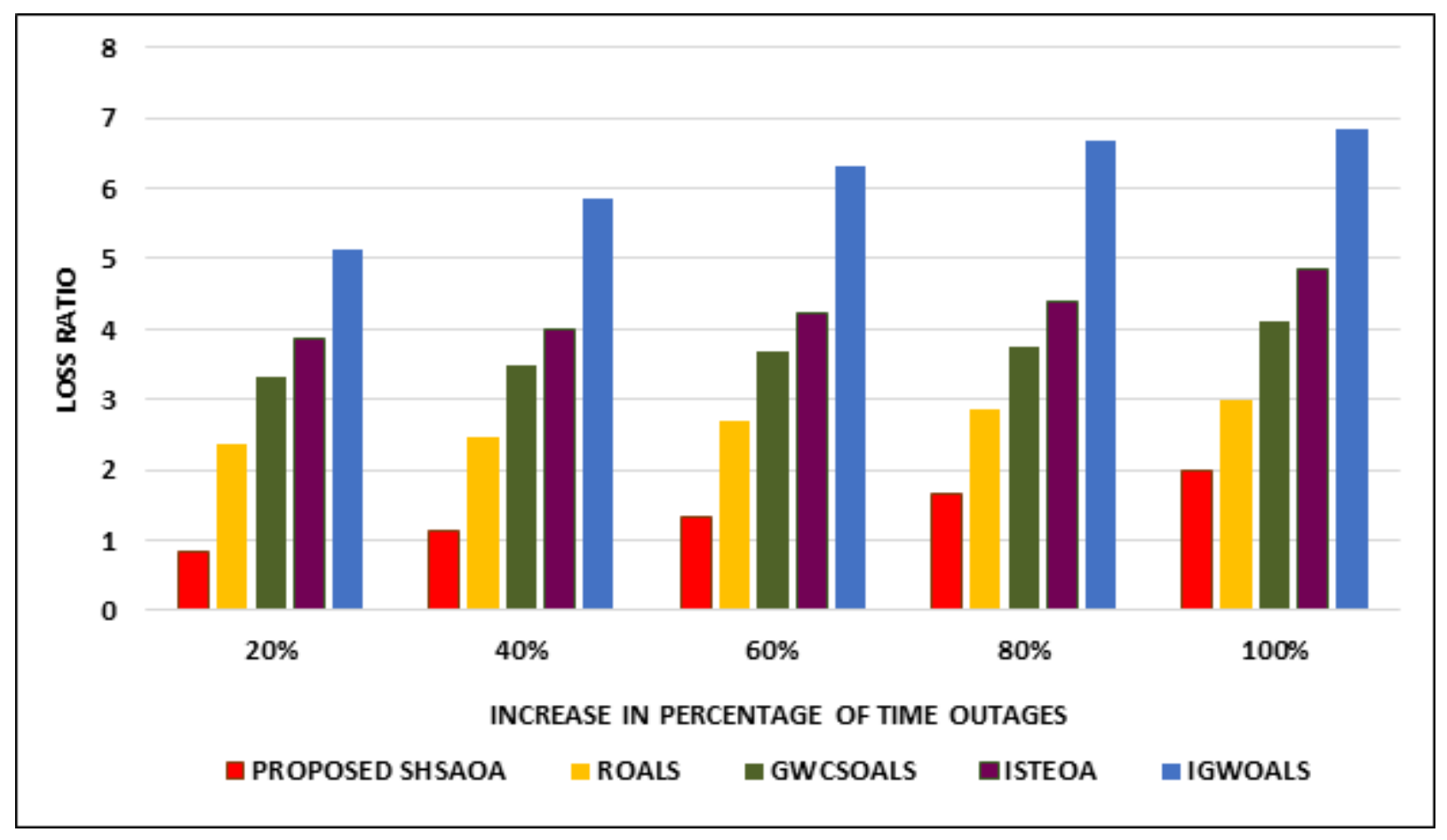

Figure 16

Proposed SHSAOA: Loss Ratio with different time outages

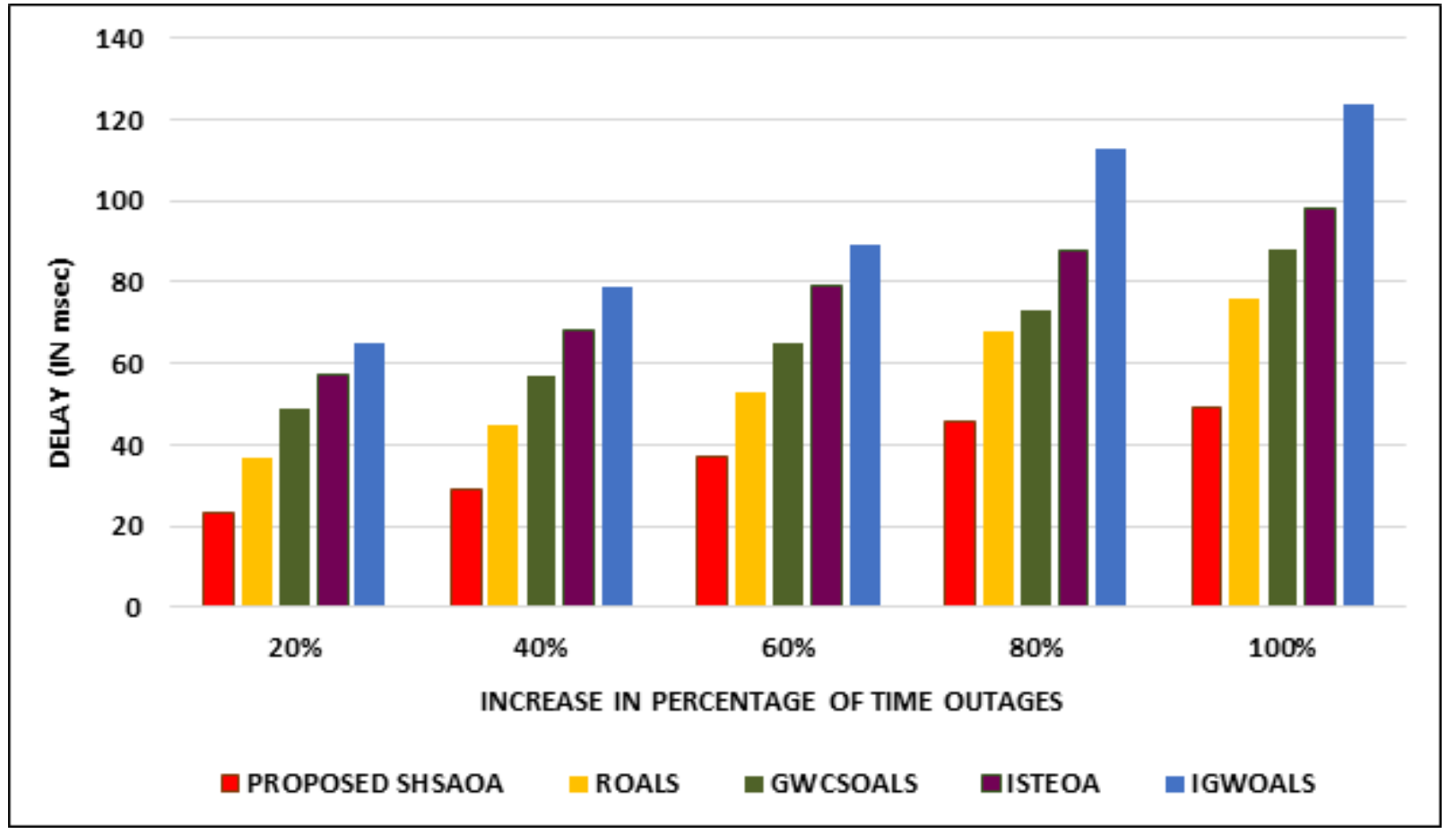

Figure 17

Proposed SHSAOA: Delay with different time outages 